\title{
Natural Language Processing Reveals Vulnerable Mental Health Support Groups and Heightened Health Anxiety on Reddit during COVID-19: An Observational Study
}

\author{
Daniel M. Low ${ }^{1,2,3^{*}}$, Laurie Rumker ${ }^{4,5,6 \dagger}$, Tanya Talkar ${ }^{1,7 \dagger}$, John Torous ${ }^{8}$, Guillermo \\ Cecchi $^{9}$, Satrajit S. Ghosh ${ }^{1,3,10}$
}

\author{
${ }^{1}$ Program in Speech and Hearing Bioscience and Technology, Harvard Medical School, Boston, MA, USA \\ ${ }^{2}$ Department of Brain and Cognitive Sciences, MIT, Cambridge, MA, USA \\ ${ }^{3}$ McGovern Institute for Brain Research, MIT, Cambridge, MA, USA \\ ${ }^{4}$ Program in Biomedical Informatics and Integrative Genomics, Harvard Medical School, Boston, MA, USA \\ ${ }^{5}$ Brigham and Women's Hospital, Boston, MA, USA \\ ${ }^{6}$ Broad Institute of MIT and Harvard, Cambridge, MA, USA \\ ${ }^{7}$ Human Health and Performance Systems, MIT Lincoln Laboratory, Lexington, MA, USA \\ ${ }^{8}$ Digital Psychiatry Division, Department of Psychiatry at Beth Israel Deaconess Medical Center, Harvard Medical \\ School, Boston, MA, USA \\ ${ }^{9}$ Thomas J. Watson Research Center, IBM, Yorktown Heights, NY, USA \\ ${ }^{10}$ Department of Otolaryngology, Harvard Medical School, Boston, MA, USA \\ * Correspondence can be addressed to Daniel M. Low, Office: 46-4033F, 43 Vassar St, Cambridge, MA 02139, \\ USA. E-mail: dlow@mit.edu \\ $\dagger$ Contributed equally
}




\begin{abstract}
Background: The COVID-19 pandemic is exerting a devastating impact on mental health, but it is not clear how people with different types of mental health problems were differentially impacted as the initial wave of cases hit. Objective: We leverage natural language processing (NLP) with the goal of characterizing changes in fifteen of the world's largest mental health support groups (e.g., $r$ /schizophrenia, $r$ /SuicideWatch, $r$ /Depression) found on the website Reddit, along with eleven non-mental health groups (e.g., r/PersonalFinance, r/conspiracy) during the initial stage of the pandemic.

Methods: We create and release the Reddit Mental Health Dataset including posts from 826,961 unique users from 2018 to 2020. Using regression, we analyze trends from 90 text-derived features such as sentiment analysis, personal pronouns, and a "guns" semantic category. Using supervised machine learning, we classify posts into their respective support group and interpret important features to understand how different problems manifest in language. We apply unsupervised methods such as topic modeling and unsupervised clustering to uncover concerns throughout Reddit before and during the pandemic.

Results: We find that the r/HealthAnxiety forum showed spikes in posts about COVID-19 early on in January, approximately two months before other support groups started posting about the pandemic. There were many features that significantly increased during COVID-19 for specific groups including the categories "economic stress", "isolation", and "home" while others such as "motion" significantly decreased. We find that support groups related to attention deficit hyperactivity disorder (ADHD), eating disorders (ED), and anxiety showed the most negative semantic change during the pandemic out of all mental health groups. Health anxiety emerged as a general theme across Reddit through independent supervised and unsupervised machine learning analyses. For instance, we provide evidence that the concerns of a diverse set of individuals are converging in this unique moment of history; we discover that the more users posted about COVID-19, the more linguistically similar (less distant) the mental health support groups became to $\mathrm{r} /$ HealthAnxiety $(\rho=-0.96, P<.001)$. Using unsupervised clustering, we find the Suicidality and Loneliness clusters more than doubled in amount of posts during the pandemic. Specifically, the support groups for borderline personality disorder and post-traumatic stress disorder became significantly associated with the Suicidality cluster. Furthermore, clusters surrounding Self-Harm and Entertainment emerged.

Conclusions: By using a broad set of NLP techniques and analyzing a baseline of pre-pandemic posts, we uncover patterns of how specific mental health problems manifest in language, identify at-risk users, and reveal the distribution of concerns across Reddit which could help provide better resources to its millions of users. We then demonstrate that textual analysis is sensitive to uncover mental health complaints as they arise in real time, identifying vulnerable groups and alarming themes during COVID-19, and thus may have utility during the ongoing pandemic and other world-changing events such as elections and protests from the present or the past.
\end{abstract}

Keywords: COVID-19; mental health; psychiatry; social media; Reddit; natural language processing; ADHD; eating disorders; anxiety; suicidality 


\section{Introduction}

The ongoing outbreak of a novel coronavirus causing the disease COVID-19 is likely to have devastating impacts on mental health as many individuals suffer from losses of income, social engagement, mobility, physical health, and uncertainty. Characterizing these impacts is critical to motivate and inform the provision of appropriate therapeutic responses. Public commentary posted to mental health support groups on the website Reddit through subfora (subreddits) captures in real-time the language used by those sharing and processing their pandemic experiences online. In this study, we apply text processing and machine learning techniques to this dataset in order to analyze COVID-19's impacts on mental health discourse, as a potential proxy for changes in mental health needs.

In the setting of a quarantine, incidence rates rise for mood disorders including Acute Stress Disorder, Post-Traumatic Stress Disorder (PTSD), Major Depressive Disorder, and Generalized Anxiety Disorder, as do rates of sub-clinical mental health deterioration [1]. Early data from the present outbreak gathered in China indicates that rates of depressive symptoms (50.4\%), anxious symptoms (44.6\%), insomnia (34\%) and general distress (71.5\%) are especially high among healthcare workers [2]. Young adults with pre-existing mental and physical health conditions indicate worsening symptoms of depression and anxiety, particularly nonbinary and female young adults [3]. Increased alcohol consumption is linked to isolation, income loss, and adjustments to living with children [4]. The scope of mental health deterioration during this pandemic provides an unprecedented need to understand how different mental health cohorts are responding to the outbreak in order to best design patient assessments and allocate resources.

Using natural language processing (NLP) to infer mental states of individuals from their social media posts is a growing field (for reviews, see [5,6]). On Reddit, individuals compose anonymous posts on 'subreddit' fora, each with a central topic. Although Reddit posts are not accompanied by formal clinical diagnoses and related covariates, this dataset offers several advantages relative to traditional mental health clinical datasets: (1) data is immediately and publicly available, (2) historical data allows for comparisons of multiple timeframes, and (3) anonymous and free-form posts create an ecological documentation of vulnerable first-person experiences.

Machine learning models have been created to classify and characterize Reddit posts originating from mental health subreddits [7-10]. Shen and Rudzicz (2017) achieved 98\% accuracy in separating posts on any of four different anxiety-related subreddits from posts on control subreddits. They utilized a combination of N-gram language modeling and Linguistic Inquiry and Word Count (LIWC) [11]. LIWC has successfully aided in detection of depression from Twitter activity with an accuracy of 70\% [12] and of bipolar disorder from Reddit posts [8]. These models typically focus on binary classification of posts with respect to a single disorder or subreddit (e.g. was the post made on $r$ /Anxiety or a control subreddit).

Visualization and analysis of topics through unsupervised machine learning methods also aid in differentiating between mental health subreddits. Using k-means clustering, analysis of themes present in $r$ /Anxiety, $r$ /Depression, and $r$ PTSD found that the $r$ /Anxiety and $r$ /PTSD subreddits shared more common terms with each other than with the $\mathrm{r} /$ Depression subreddit [13].

Reddit can also help monitor discussions around public health subjects. Utilizing Latent Dirichlet Allocation (LDA), one study identified that Reddit discussions of Ebola focused on health education through posts about best practices and the implications of public health events [14]. Combined with an analysis of linguistic changes within mental health subreddits, topic analysis can provide insights into the issues surrounding users during public health crises.

In this paper, we (1) analyze changes and trends in language features during the pandemic, (2) develop a machine learning model that successfully classifies posts as originating from a particular mental health subreddit, (3) 
understand concerns shared across Reddit independent of subreddit of origin using unsupervised machine learning methods, and (4) assess whether subreddits are becoming more or less similar using supervised dimensionality reduction. We expect that quantifying differences between pre- and mid-pandemic post content and characterizing discussions in the r/COVID19 support group will yield valuable insight into the impact of COVID-19 on mental health and help deploy treatment more effectively.

\section{Methods}

\subsection{Reddit Mental Health Dataset}

Reddit users

Demographic information per subreddit is unavailable, but Reddit users collectively are predominantly American (49.9\%), male (67\%), and young (22\% 18-29yo; 14\% 30-49yo) [15,16].

\section{Data downloading \& preprocessing}

Data was downloaded using the pushshift API [17]. Posts were extracted from fifteen subreddits focused on specific mental health communities (r/EDAnonymous, $r$ /addiction, r/alcoholism, r/adhd, r/anxiety, r/autism, r/BipolarReddit, $\mathrm{r} / \mathrm{bpd}, \mathrm{r} /$ depression, $\mathrm{r} /$ healthanxiety, r/lonely, r/ptsd, r/schizophrenia, $\mathrm{r} /$ socialanxiety, and $\mathrm{r} /$ SuicideWatch), two broad mental health subreddits ( $\mathrm{r}$ /mentalhealth, $\mathrm{r} / \mathrm{COVID} 19$ support), and 11 non-mental health subreddits ( $\mathrm{r} /$ conspiracy, $\mathrm{r} /$ divorce, $\mathrm{r}$ /fitness, r/guns, r/jokes, r/legaladvice, r/meditation, $r$ /parenting, $r$ /personalfinance, $r /$ relationships, $\mathrm{r} /$ teaching). Details of data and preprocessing are provided in Supplementary Methods 1.1.

\section{Feature extraction}

The following features were extracted from posts: LIWC (62), sentiment analysis (4), basic word and syllable counts (8), punctuation (1), readability metrics (9), TF-IDF ngrams (256-1024), and manually built lexicons about suicidality (1), economic stress (1), isolation (1), substance use (1), domestic stress (1), guns (1). Trend Analysis used all features except TF-IDF, classification and supervised dimensionality reduction used all features, as did unsupervised clustering, and LDA used TF-IDF features. See the Supplementary Methods 1.2 for more details.

\subsection{Classification and Feature Importance} Training and testing

Binary classification was performed on each of the 15 specific mental health subreddits versus a control group $(\mathrm{N}=$ 2700 each) made of a random sample of the remaining subreddits (first balanced to assure subreddits were equally represented). Only one post per user was used to avoid overfitting. 80-20 train-test split was used. Two additional test sets were built to test the pre-pandemic model. One included mid-pandemic (from 2020-03-11 to 2020-04-20) (mean $\mathrm{N}=504$ posts combined; $\mathrm{SD}=54.3$ ) to measure potential dataset shift. A second test set was composed of posts from $\mathrm{r} / \mathrm{COVID} 19$ support and a control group $(\mathrm{N}=1574$ combined) to measure how they will be classified by our pre-pandemic model. Weighted F1 was used to measure performance.

\section{Models}

Our goal was to use the model with lowest complexity in order to determine feature importance more directly as long as the model is not considerably outperformed by more complex models. We tested three linear models (stochastic gradient descent linear classifier (SGD) with L1 penalty, SGD with elastic net penalty, linear support vector machine) along with two more complex tree ensemble classifiers (extra trees, gradient boosting trees). 


\subsection{Trend Analysis}

We first tracked COVID-19 related tokens (see Supplementary Table S1) from 01/01/2020 to 04/20/2020 across mental health subreddits. We compare this to the confirmed COVID-19 cases with data obtained from ourworldindata.org/covid-cases (source: European CDC). We then grouped data every two days from 01/01 to 04/20 for every year. For each of the 90 features and 28 subreddits, we fit a linear regression for the average feature value at each time point as a function of time. We apply the Benjamini-Hochberg procedure for multiple hypothesis testing correction with $\alpha=0.05$. We define change for each feature and subreddit as the slope $\times R^{2}$ (i.e., the rate of change weighed by the goodness of fit). We test the amount of absolute change between years by using a Mann-Whitney $\mathrm{U}$ test.

\subsection{Unsupervised Clustering}

Pre-pandemic posts made in 2019 from 15 mental health subreddits were downsampled to balanced representation (1500 posts/subreddit). The feature set for these posts described above, including 1024 TF-IDF ngrams, was reduced to 30 PCA components. scikit-learn's SpectralClustering function was used to identify $k=20$ clusters, using default parameters apart from a Nearest-Neighbors-based $(n=10)$ affinity matrix. (Selection of k described in Supplementary Fig. S8.) Wilcoxon rank-sum tests (with Bonferroni correction) identified cluster-characteristic features for annotating each cluster with a defining theme. Cluster annotation validity was verified through post review. Hypergeometric tests (with Bonferroni correction) identified enrichment of posts from particular clusters on particular subreddits. Posts from the mid-pandemic dataset were processed through the same pipeline. The resulting clusters were compared on the basis of cluster-characteristic features to clusters defined in the pre-pandemic dataset, and the vast majority of clusters were found to have a close match pairing in each time window, as illustrated in Supplemental Table S4.

\subsection{Topic Modeling with Latent Dirichlet Allocation}

The gensim library was used to perform LDA model estimation. To balance across subreddits, 2700 pre-pandemic posts from 2019 were sampled from each of the 15 focused mental health subreddits to form our model. Multiple models were created to assess topic stability. A final pre-pandemic model with 10 topics was chosen to ensure distinct and important topics. A separate model was created with 10 topics using 1300 posts from each mid-pandemic mental health subreddits, spanning 2020/01/01 to 2020/04/20 to assess any shift in topics. Models were then applied to all subreddits to assess the distribution of the posts across the topics. Models were applied to mid-pandemic posts between 2020/03/16 and 2020/04/20 to capture topic distributions during the acute phase of the pandemic. Comparison of topic distributions between pre- and mid-pandemic posts was done with a two-sided Wilcoxon signed-rank test with the Benjamini-Hochberg procedure $(\alpha=0.05)$ to test whether the incidence of these topics changed across subreddits as a result of the pandemic. Details are provided in Supplementary Methods 1.3.

\subsection{Measuring Similarity Between Subreddits over Time with Supervised Dimensionality Reduction}

With the goal of measuring the similarity between the 15 mental health subreddits as COVID-19 spread, we first reduced posts' $346 \mathrm{D}$ feature vectors to $2 \mathrm{D}$ using supervised UMAP, a nonlinear dimensionality reduction technique that better preserves global structure in comparison to other methods such as t-SNE [18]. We then measured the asymmetric Hausdorff distance between subreddits as time progresses to estimate which subreddits are becoming more similar (i.e., less distant) to each other. We balanced subreddits to 1300 posts each spanning from 2020/01/01 to 2020/04/20 grouped in 15-day time windows. This subsampling and dimensionality reduction was repeated for 50 bootstrapping samples and we took the median distance value. See Supplementary Methods 1.4 for a test of the precision of this method on 2019 data. 


\section{Results}

\subsection{Classification and Feature Importance}

Models all performed similarly with mean weighted F1 scores between 0.798 to 0.857 (see Supplementary Table $\mathrm{S} 1)$. The SGD L1 $(\mathrm{F} 1=0.851)$ was chosen for further analysis given it had the lowest model complexity. Important features are available in Table 1. We then applied this model to mid-pandemic data (2020/03/11 to 2020/03/-20) and performance changed in several classes (see Supplementary Table S3). These binary classification models were applied to r/COVID19_support posts in order to characterize them psychologically (see Figure 4).

\subsection{Trend Analysis}

See Figure 1 for the proportion of posts about COVID-19 in each support group. For a measure of how much of each post was about COVID-19, see Supplementary Figure S3. For an example of how trends were computed for a single feature and subreddit, see Supplementary Fig. S4. Figure 2 shows a subset of the trends and most negative semantic change (i.e., sum of change in negative semantic features). Throughout many subreddits, we found significant increases in the use of tokens related to isolation (e.g., 'lonely', "can't see anyone", 'quarantine'), economic stress (e.g., 'rent', 'debt', 'pay the bills'), home ('fridge', 'pet', 'lease'), and anxiety ('afraid', 'scary', 'uncertain') and a decrease in the lexicon related to motion (e.g., 'walk', 'visit', 'travel'), all consistent with the type of changes many are facing during the ongoing pandemic. See Supplementary Figures S5-S7 for full results (90 features in 28 subreddits). See Table 2 for examples of posts that score high on important features and display risky behavior.

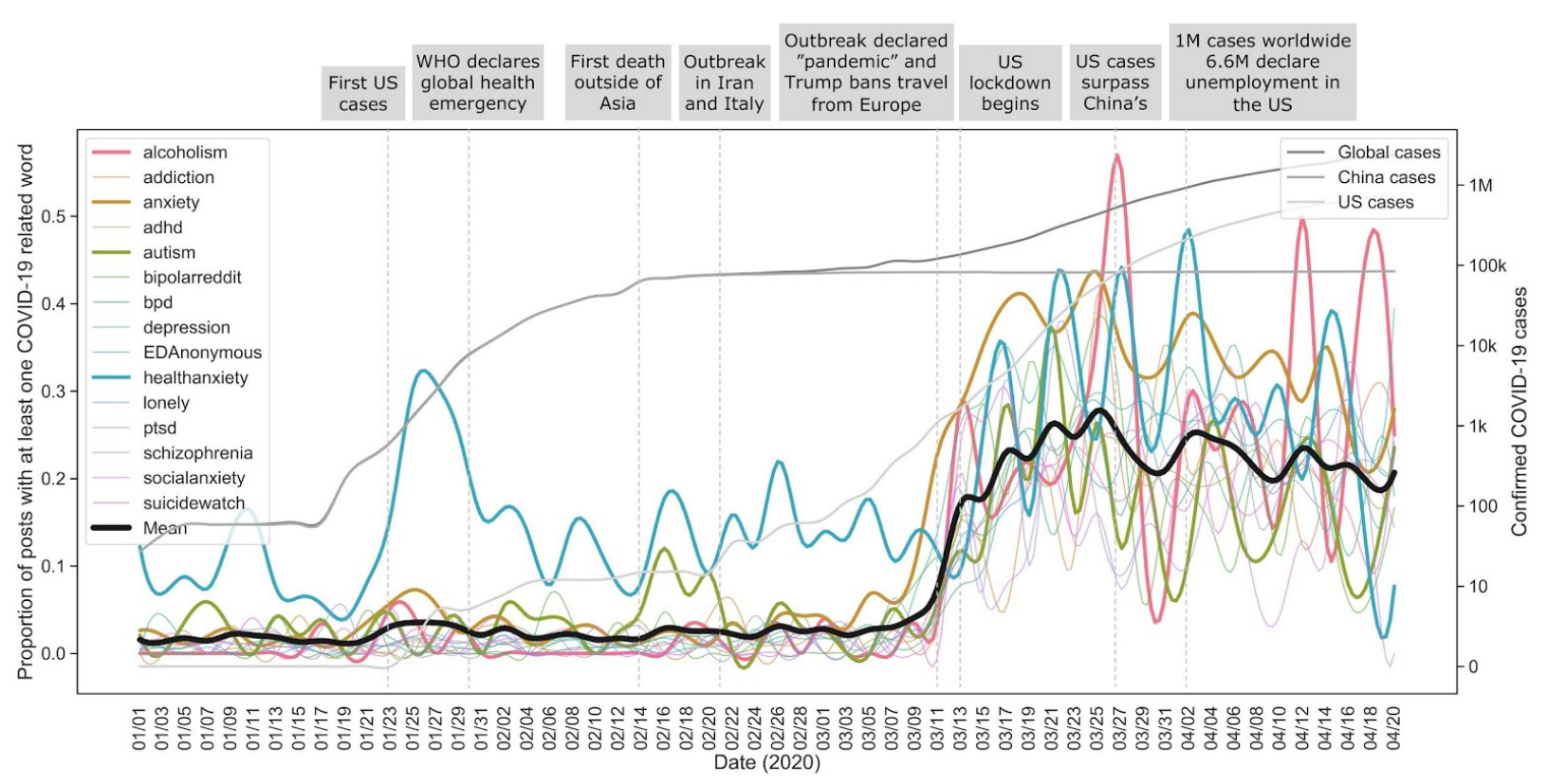

Figure 1: Mention of COVID-19 related words across mental health support groups. Timeline landmarks are chosen from NBC News timeline given U.S. users are the most prevalent across Reddit. Global, China, and U.S. confirmed COVID-19 cases are displayed. The overall acute rise in COVID-19 related words occurs on March 11. The correlation between mean proportion of COVID-19 related posts and global COVID-19 cases is $\rho=0.83$ $(P<.001)$. The health anxiety subreddit has a large increase in COVID-19 related posts almost two months before the general increase. $r$ /alcoholism has the most amount of posts related to COVID-19 on March 27. bpd: borderline personality disorder; EDAnonymous: Eating Disorders Anonymous; ptsd: post-traumatic stress disorder. 


\begin{tabular}{|c|c|c|}
\hline Subreddit & Positive coefficients & Negative coefficients \\
\hline r/EDAnonymous & $\begin{array}{l}\text { ed, restrict, purg, bing, calori, LIWC ingestion, } \\
\text { fast, recoveri, eat, ate }\end{array}$ & $\begin{array}{l}\text { bpd, anxieti, addict, diagnos, drug, substance } \\
\text { use lexicon, ptsd, LIWC work, LIWC health, } \\
\text { med }\end{array}$ \\
\hline r/addiction & $\begin{array}{l}\text { addict, clean, smoke, rehab, sober, drug, weed, } \\
\text { relaps, use, guns lexicon }\end{array}$ & $\begin{array}{l}\text { bpd, diagnos, ptsd, adhd, therapi, isolation } \\
\text { lexicon, LIWC hear, therapist, post, LIWC } \\
\text { work }\end{array}$ \\
\hline r/adhd & $\begin{array}{l}\text { adhd, adderal, add, vyvans, focu, forget, final, } \\
\text { LIWC work, medic, came }\end{array}$ & $\begin{array}{l}\text { bpd, ptsd, hurt, therapi, guess, suicidality } \\
\text { lexicon, fear, bodi, suicid, pain }\end{array}$ \\
\hline r/alcoholism & $\begin{array}{l}\text { sober, alcohol, drink, withdraw, drunk, LIWC } \\
\text { nonfluencies, drank, meet, beer }\end{array}$ & $\begin{array}{l}\text { drug, weight, therapi, adhd, medic, isolation } \\
\text { lexicon, notic, attack, dure, addict }\end{array}$ \\
\hline r/anxiety & $\begin{array}{l}\text { anxieti, wa dead, LIWC negative emotion, } \\
\text { LIWC money, anxiou, LIWC motion, LIWC } \\
\text { numbers, panic attack, anxious }\end{array}$ & $\begin{array}{l}\text { ptsd, bpd, adhd, LIWC ingestion, LIWC } \\
\text { articles article, addict, kill, substance use } \\
\text { lexicon, LIWC body, social }\end{array}$ \\
\hline r/autism & $\begin{array}{l}\text { autism, autist, spectrum, son, game, diagnos, } \\
\text { function, diagnosi, explain, interest }\end{array}$ & $\begin{array}{l}\text { ptsd, bpd, addict, LIWC health, disord, adhd, } \\
\text { med, 2, stay, guns lexicon }\end{array}$ \\
\hline r/BipolarReddit & $\begin{array}{l}\text { bipolar, manic, mania, lithium, mood, episod, } \\
\text { psychiatrist, hospit, LIWC money, med }\end{array}$ & $\begin{array}{l}\text { adhd, addict, bpd, ptsd, LIWC ingestion, LIWC } \\
\text { anxiety, LIWC work, automated readability } \\
\text { index, LIWC future tense }\end{array}$ \\
\hline $\mathrm{r} / \mathrm{bpd}$ & $\begin{array}{l}\text { bpd, fp, LIWC numbers, LIWC inclusive, } \\
\text { LIWC negative emotion, LIWC sadness, bad, } \\
\text { drug, LIWC affective processes, feel }\end{array}$ & $\begin{array}{l}\text { ptsd, adhd, weight, LIWC articles article, } \\
\text { LIWC health, addict, LIWC 1st pers, anxious, } \\
\text { food, isolation lexicon }\end{array}$ \\
\hline $\mathrm{r} /$ depression & $\begin{array}{l}\text { depress, LIWC sadness, LIWC negations, } \\
\text { gunning fog index, LIWC positive emotion, } \\
\text { LIWC family, cri, LIWC feel, bed, LIWC total } \\
\text { pronouns }\end{array}$ & $\begin{array}{l}\text { bpd, symptom, ptsd, adhd, food, isolation } \\
\text { lexicon, LIWC conjunctions, diagnos, addict, } n \\
\text { sents }\end{array}$ \\
\hline $\mathrm{r} /$ healthanxiety & $\begin{array}{l}\text { cancer, LIWC biological, health anxieti, LIWC } \\
\text { health, health, LIWC body, test, fine, LIWC } \\
\text { assent, googl }\end{array}$ & $\begin{array}{l}\text { ptsd, adhd, bpd, addict, emot, disord, LIWC 3rd } \\
\text { pers, LIWC social processes, social, mental }\end{array}$ \\
\hline r/lonely & $\begin{array}{l}\text { lone, loneli, isolation lexicon, messag, LIWC } \\
\text { certainty, friend, girl, LIWC positive emotion, } \\
\text { sit, LIWC religion }\end{array}$ & $\begin{array}{l}\text { LIWC anxiety, ptsd, addict, bpd, suicidality } \\
\text { lexicon, symptom, therapist, abus, suicid, med }\end{array}$ \\
\hline $\mathrm{r} / \mathrm{ptsd}$ & $\begin{array}{l}\text { ptsd, trauma, flashback, trigger, nightmar, } \\
\text { sexual, domestic stress lexicon, abus, tire, guns } \\
\text { lexicon }\end{array}$ & $\begin{array}{l}\text { bpd, addict, drink, adhd, LIWC health, isolation } \\
\text { lexicon, LIWC work, disord, LIWC certainty, } \\
\text { LIWC sadness }\end{array}$ \\
\hline r/schizophrenia & $\begin{array}{l}\text { schizophrenia, hallucin, delus, schizophren, } \\
\text { voic, paranoid, LIWC religion, hospit, ill, } \\
\text { LIWC tentative }\end{array}$ & $\begin{array}{l}\text { adhd, ptsd, bpd, addict, abus, flesch kincaid } \\
\text { grade level, isolation lexicon, LIWC body, } \\
\text { LIWC biological, LIWC health }\end{array}$ \\
\hline r/socialanxiety & $\begin{array}{l}\text { social anxieti, nervou, walk, awkward, girl, } \\
\text { group, convers, speak, face, anxieti }\end{array}$ & $\begin{array}{l}\text { bpd, adhd, ptsd, LIWC health, addict, diagnos, } \\
\text { suicid, LIWC sadness, support }\end{array}$ \\
\hline r/SuicideWatch & $\begin{array}{l}\text { suicidality lexicon, suicid, LIWC negations, } \\
\text { death, kill, want die, LIWC sadness, LIWC } \\
\text { friends, LIWC money, plan }\end{array}$ & $\begin{array}{l}\text { bpd, symptom, anxious, substance use lexicon, } \\
\text { usual, LIWC 2nd pers, smog index, isolation } \\
\text { lexicon, weight, attack }\end{array}$ \\
\hline
\end{tabular}

Table 1. Important features for classification (ranked). Their presence makes it more (positive) or less (negative) likely the classifier will predict the subreddit. Individual word stems are obtained from TF-IDF. 
A

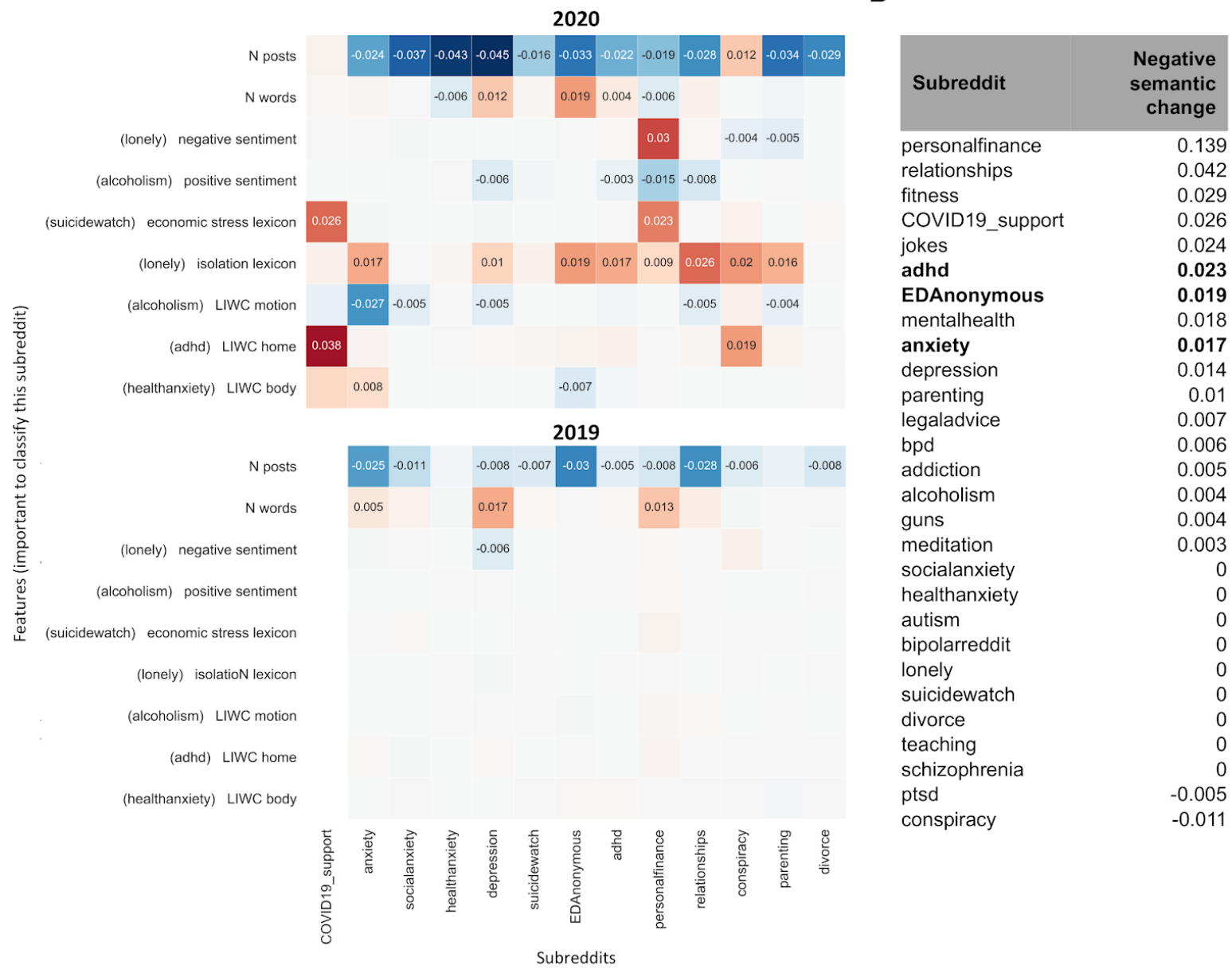

Figure 2: Trend analysis of linguistic features over time. A) Significant change in average feature values from January to April 2020 and 2019 across subreddits. COVID19 support subreddit was created in 2020 and therefore does not appear in 2019 (see Supplementary Figs. S5, S6, S7 for full results with 90 features and 28 subreddits from 2018 to 2020; see Table 2 for examples of posts). Features important for classifying a subreddit are added to the $\mathrm{y}$-axis. Change is defined by slope $\times \mathrm{R}^{2}$ (i.e., increases have positive slope and tend towards red, decreases have negative slope and tend towards blue, see examples in Supplementary Fig. S4). Significant trends after multiple comparison correction on full results are displayed. There is significantly more absolute change in 2020 than in 2019 $(P<.001)$ and $2018(P<.001)$. B) Rank of subreddits by amount of negative semantic change throughout COVID-19 $(01 / 01 / 2020-04 / 20 / 2020)$ across significant full results. In bold are the mental health subreddits with the most negative semantic change using the following features with emotional valence: negative sentiment; the lexicons about economic stress, isolation, substance use, guns, domestic stress, and suicidality; LIWC measures of anger, anxiety, death, negations, negative emotion, and sadness; and three positive features inversely weighed, compound sentiment, positive sentiment and positive emotion. $r$ ptsd and $r$ conspiracy decreased in negative semantic features. 


\begin{tabular}{|c|c|}
\hline Feature & Post \\
\hline $\begin{array}{l}\text { COVID-19 } \\
\text { lexicon }\end{array}$ & $\begin{array}{l}\text { r/healthanxiety (01/19): I've been seeing a lot of news stories today about an incurable, lethal } \\
\text { disease originating in China spreading to other countries. I know the media often over exaggerates } \\
\text { things like this, but WHO is debating whether or not to declare this an international emergency. } \\
\text { Nothing triggers my anxiety worse than things like this, if anyone has any advice on how to } \\
\text { cope/can inform me on it I would really appreciate it! }\end{array}$ \\
\hline \begin{tabular}{|l} 
LIWC \\
achievement
\end{tabular} & $\begin{array}{l}\text { r/addiction (03/20): Anyone else relapse during quarantine? Looking for others who are going } \\
\text { through this and would like to swap experience, strength and hope? (...) all my meetings have been } \\
\text { canceled }\end{array}$ \\
\hline $\begin{array}{l}\text { LIWC } \\
\text { affective } \\
\text { processes }\end{array}$ & $\begin{array}{l}\text { r/bpd (03/27): (...) I posted this in another group but I'm really struggling right now. I really wish I } \\
\text { could get into a DBT group but now I'm so confused with all of this virus stuff. Does anyone have } \\
\text { effective tips for dealing with splitting? Sometimes I will be upset with my S/O for hours or days } \\
\text { and I don't even know why at a certain point. I get so manipulative and I really hate it. Does anyone } \\
\text { else get like this? }\end{array}$ \\
\hline $\begin{array}{l}\text { LIWC } \\
\text { ingestion }\end{array}$ & $\begin{array}{l}\text { r/EDAnonymous (03/19): Quarantine is slowly chipping away at my recovery. I can't help but feel } \\
\text { like this is the perfect time to fast or restrict since no one's monitoring my meals. Nobody at school } \\
\text { noticing. I'm skipping lunch for the } 4 \text { th day in a row or only eating celery. I was doing so well!!! } \\
(\ldots)\end{array}$ \\
\hline $\begin{array}{l}\text { LIWC money } \\
\text { and guns } \\
\text { lexicon }\end{array}$ & $\begin{array}{l}\mathbf{r} / \text { SuicideWatch (03/29): Suicide is too expensive with increasing gun prices I've been watching } \\
\text { gun deals to see if I could afford something that was quick and surefire, but covid has made guns } \\
\text { more expensive with greater wait times. }\end{array}$ \\
\hline $\begin{array}{l}\text { LIWC } \\
\text { negations }\end{array}$ & $\begin{array}{l}\text { r/depression (03/21): I can't do it If things don't get back to normal in a few weeks I want to kill } \\
\text { myself. The only thing I was excited about was starting a new job and I can't anymore because of } \\
\text { all the coronavirus shut downs. I'm going crazy I don't have anyone to talk to and I just can't do } \\
\text { this anymore }\end{array}$ \\
\hline LIWC see & $\begin{array}{l}\text { r/socialanxiety (04/13): Now that everyone is wearing face masks, I suddenly don't have trouble } \\
\text { making eye contact with people anymore. I might keep wearing a mask out in public after the } \\
\text { quarantine is over. I really like the confidence it gives me. I don't have to worry about what my } \\
\text { facial expression is or looks like, I don't have to worry about smiling, everyone looks the same } \\
\text { around me. }\end{array}$ \\
\hline $\begin{array}{l}\text { Economic } \\
\text { stress } \\
\text { lexicon }\end{array}$ & $\begin{array}{l}\text { r/COVID19_support (03/10): Would I be allowed a temporary paid Leave of Absence by my } \\
\text { psychiatrist from my unionized supermarket due to COVID19? It's causing me major anxiety, } \\
\text { everyone else is calm but it sadly compromises my calmness. I am diagnosed with schizoaffective } \\
\text { disorder/bipolar. There's only one confirmed case in my county, but still. School for me, may be } \\
\text { canceled. How do I handle working my part time job? }\end{array}$ \\
\hline $\begin{array}{l}\text { Negative } \\
\text { sentiment }\end{array}$ & $\begin{array}{l}\text { r/adhd (03/20): Drug interactions with COVID-19?? I just read about methylphenidate being } \\
\text { directly related to a lower white blood cell count/ worse immune system.. AND it can cause high } \\
\text { blood pressure. I'm hyper-focused on this virus and i'm terrified that Concerta is putting me more } \\
\text { at risk. Anyone else in the same boat? }\end{array}$ \\
\hline LIWC health & $\begin{array}{l}\text { r/conspiracy }(\mathbf{0 3} / \mathbf{1 3}):(\ldots) \text { How has the coronavirus been treated? Iodine and vitamin C. How has } \\
\text { radiation sickness been treated? Iodine and vitamin C. China and Italy lead the world in radioactive } \\
5 \mathrm{G} \text { cell towers. } 5 \mathrm{G} \text { operates on the same frequency and wavelength as military grade weapon } \\
\text { technology. Connect the dots. }\end{array}$ \\
\hline
\end{tabular}

Table 2. Example of posts that score high on a subset of important features. The $\mathrm{r} /$ healthanxiety post occurs in January when other subreddits have relatively no activity about the coronavirus (see Figure 1). A positive reaction from a r/socialanxiety user is included. More examples are included in the shared dataset. 


\subsection{Unsupervised Analysis}

\section{Unsupervised Clustering Uncovers Post Language Themes}

Clustering of pre-pandemic posts (Figure 3, Supplementary Figure S8) highlights that language use across subreddits represents a continuum but contains meaningful axes of variation reflected by the proximity of clusters for closely related conversation topics. Cluster-distinguishing terms (Supplementary Table S4) used to assign annotations reveal that some clusters are characterized by discussion of particular mental health concerns (e.g., "Suicidality") while others are characterized by post tone (e.g., "Seeking Normalization").

We identified 47 cluster-subreddit pairings (Figure 3B) for which posts from the given cluster were enriched on the given subreddit. Expected pairings were recapitulated and additional associations of interest were revealed. For example, r/addiction is enriched for posts from the "Substance Use Alcohol" and "Substance Use Marijuana" clusters, as expected. It is also one of few subreddits enriched for posts from the "Resources" and "Seeking Advice" clusters. Though cluster-subreddit associations are present, every cluster contains posts from $\geq 12$ subreddits, emphasizing the value of clustering to define categories that span subreddits. Clusters defined by tone and unspecified clusters contain the greatest diversity of post representation across subreddits, quantified by Shannon Index (Supplementary Table S5). The distribution of posts across subreddits within four example clusters in Supplementary Figure S9 illustrate the subreddits with greatest representation in the Medication cluster (r/adhd, $\mathrm{r} /$ BipolarReddit, r/schizophrenia, $\mathrm{r} /$ anxiety), the Pleading cluster (r/SuicideWatch, r/lonely, r/depression, r/addiction), the Social Anxiety cluster ( $\mathrm{r} /$ socialanxiety, $\mathrm{r} /$ anxiety, $\mathrm{r} /$ autism), and the Suicidality cluster $(\mathrm{r} /$ SuicideWatch, $\mathrm{r} /$ depression, $\mathrm{r} / \mathrm{bpd}$ )

\section{LDA Topic Modeling on Multiple Timeframes}

Figure 4 shows the distribution of posts in mid-pandemic mental health subreddits over the 10 topics extracted using LDA on pre-pandemic mental health subreddits as well as the 10 topics extracted using LDA on the mid-pandemic mental health subreddits. The topic number was chosen to include distinct yet important topics. Topics extracted from the pre-pandemic LDA model (x-axis of Figure 4A, and Supplementary Table S6) largely matched the expected topics from the pre-pandemic subreddits. Topics emerged related to "Alcoholism and Addiction", "Health Anxiety", "Alcoholism and Eating Disorder", "Schizophrenia", and "ADHD and Autism", corresponding to specific subreddits. The ADHD and autism token words were also combined into the same topic with tokens related to school and work, and did not separate even in models with a larger number of topics. More general topics such as "Social Interaction", "Life", and "Mental Health Help" also emerged, which captured common topics in subreddits such as $\mathrm{r} /$ SuicideWatch, $r$ /depression, $r$ /lonely and $r /$ mentalhealth. Compared to the pre-pandemic topic model, the mid-pandemic LDA model splits the autism and ADHD tokens, includes a topic on family, and also includes a topic with a PTSD token. Supplementary Materials contain the distribution of pre-pandemic model topics across mental health subreddits (Supplementary Figure S10) and across non-mental health subreddits (Supplementary Figure S11) in both pre- and mid-pandemic timeframes. Wilcoxon signed-rank tests of distributions of the pre- and mid-pandemic posts over pre-pandemic model topics implies there was an increase in the "Health Anxiety" $(P=.008)$ topic and in the "Life" topic $(P=.014)$, as well as a decrease in the "Alcoholism and Addiction" topic $(P=.004)$ while the remainder of the topics showed no significant change in distribution after multiple comparison correction. 
A

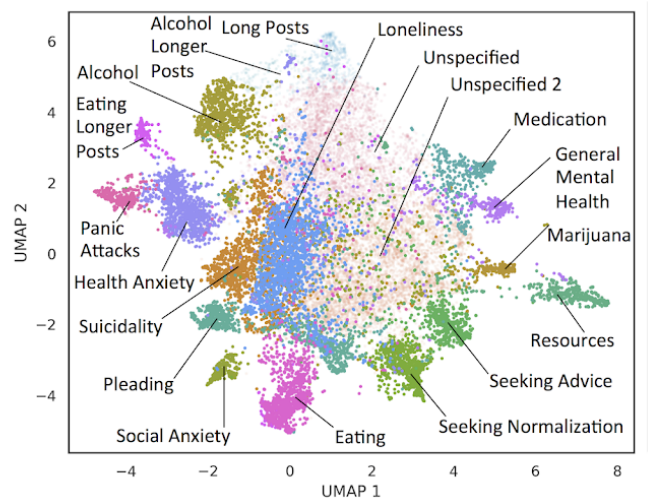

B

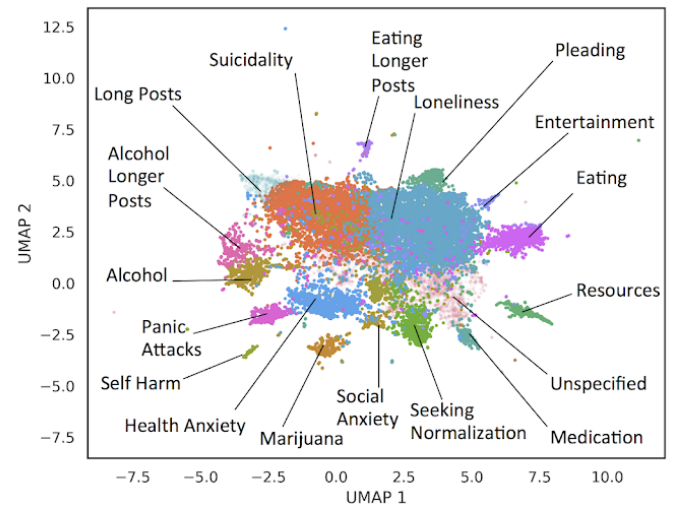

C

\begin{tabular}{|l|l|l|}
\hline \multicolumn{1}{|c|}{ Subreddit } & \multicolumn{1}{|c|}{ Enriched Clusters, Pre-Pandemic } & \multicolumn{1}{c|}{ Enriched Clusters, Mid-Pandemic } \\
\hline EDAnonymous & Eating, Eating Longer Posts & Eating, Eating Longer Posts \\
\hline addiction & Alcohol, Marijuana, Resources, Seeking Advice, Alcohol Longer Posts & Alcohol, Marijuana, Resources, Alcohol Longer Posts, Long Posts \\
\hline adhd & Medication, Unspecified, Unspecified 2 & Medication, Unspecified \\
\hline alcoholism & Alcohol, Alcohol Longer Posts & Alcohol, Alcohol Longer Posts \\
\hline anxiety & Panic Attacks, Medication, Seeking Normalization & Panic Attacks, Health Anxiety, Social Anxiety \\
\hline autism & Seeking Advice, Resources, Unspecified, Unspecified 2 & Entertainment, Seeking Normalization, Resources, Unspecified \\
\hline bipolarreddit & Medication, General Mental Health, Unspecified, Unspecified 2 & Medication, Unspecified \\
\hline bpd & General Mental Health, Loneliness, Seeking Normalization, Unspecified 2 & Long Posts, Seeking Normalization, Self Harm, Suicidality \\
\hline depression & Loneliness, Suicidality, Pleading, Unspecified 2 & Loneliness, Suicidality, Pleading \\
\hline healthanxiety & Panic Attacks, Health Anxiety, Resources & Panic Attacks, Health Anxiety \\
\hline lonely & Loneliness, Pleading & Loneliness, Entertainment \\
\hline ptsd & Panic Attacks, Seeking Advice, Unspecified, Unspecified 2 & Panic Attacks, Long Posts, Suicidality \\
\hline schizophrenia & General Mental Health, Medication, Unspecified & Unspecified \\
\hline socialanxiety & Loneliness, Social Anxiety & Seeking Normalization, Social Anxiety \\
\hline suicidewatch & Pleading, Suicidality & Loneliness, Suicidality, Self Harm, Long Posts, Pleading \\
\hline
\end{tabular}

Figure 3: Unsupervised clustering reveals post groupings with representation across mental health subreddits. A) Unsupervised clustering of pre-pandemic (year 2019) posts from 15 mental health subreddits, presented in 2D UMAP space. Posts in two thematically-related, adjacent clusters were collapsed into a single "Resources" cluster. Three clusters could not be assigned identifiable themes. Two of these - annotated as "Unspecified"-were the largest clusters in the dataset, containing 6329 and 4272 total posts, respectively, while the next largest cluster contained 1620 posts. The other cluster without an identifiable theme was characterized by very long posts. This "Long Posts" cluster had an average post length of 886 words, while the cluster with the next most lengthy posts had an average post length of 554 words. As a result, this "Long Posts" cluster had an overwhelming number of cluster-characteristic text features, which made any core linguistic theme poorly discernible. The identified clusters were not an approximation of post subreddit of origin, as demonstrated by several metrics quantifying the lack of correspondence between cluster labels and post subreddit of origin: Homogeneity (0.20), Completeness (0.22), V-measure (0.21), and Adjusted Rand-Index (0.08). B) Unsupervised clustering of mid-pandemic posts using the same process resulted primarily in replication of cluster annotations observed in the pre-pandemic data, with a few clusters (e.g., Seeking Advice) detected only in the pre-pandemic clustering and a few (e.g., Entertainment) detected only in the mid-pandemic clustering. Two clusters increased notably in size in the mid-pandemic clustering: Suicidality (204\% increase in number of posts) and Loneliness (233\% increase in number of posts). C) Enrichment of clusters on mental health subreddits during the pre-pandemic period and the mid-pandemic period, using clusters detected during each time period, respectively. Associations were assessed with hypergeometric tests, and those displayed here passed strict Bonferroni correction for multiple hypothesis testing. Associations present only for the pre-pandemic or only for the mid-pandemic time period are shown in bold. 
A

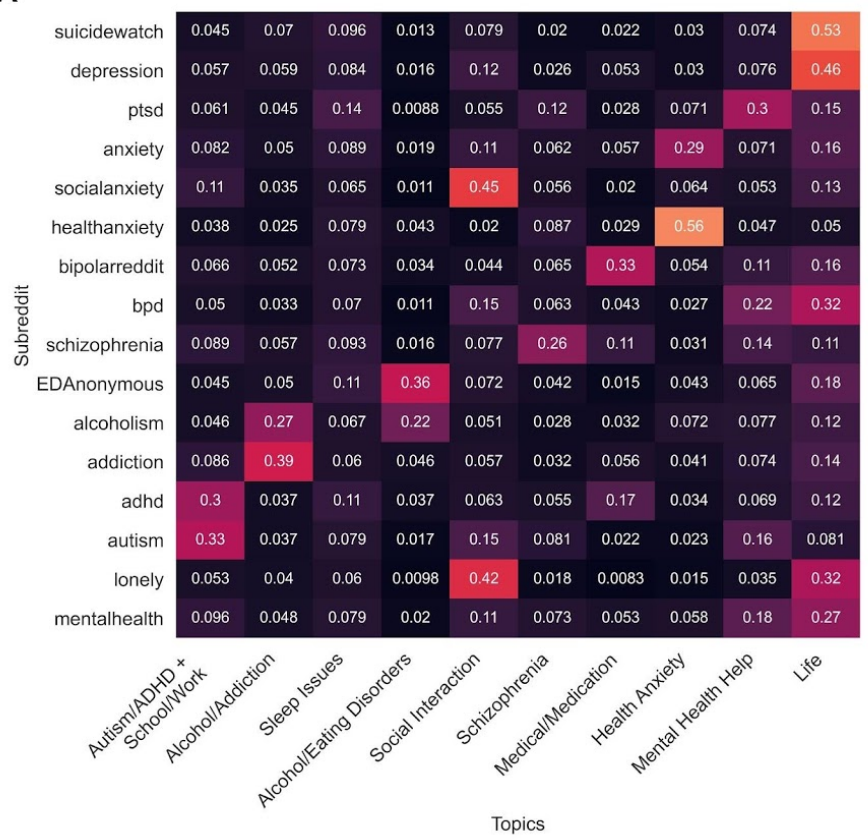

B

\begin{tabular}{|l|l|}
\hline Mid-Pandemic Topics & Words \\
\hline Health Anxiety & $\begin{array}{l}\text { anxieti, feel, go, take, doctor, panic, } \\
\text { start, week, attack, pain }\end{array}$ \\
\hline Autism/Social & $\begin{array}{l}\text { peopl, autism, autist, think, know, } \\
\text { thing, social, help, talk, look }\end{array}$ \\
\hline ADHD/Diagnosis & $\begin{array}{l}\text { adhd, diagnos, medic, help, } \\
\text { diagnosi, read, bipolar, experi, } \\
\text { symptom, take }\end{array}$ \\
\hline Work/School/Home & $\begin{array}{l}\text { work, school, time, year, go, college, } \\
\text { home, start, class, life }\end{array}$ \\
\hline Eating Disorder & $\begin{array}{l}\text { eat, weight, food, gain, lose, bing, } \\
\text { feel, fuck, look, want }\end{array}$ \\
\hline Alcohol/Addiction & $\begin{array}{l}\text { drink, alcohol, addict, time, year, go, } \\
\text { drug, stop, know, smoke }\end{array}$ \\
\hline Family & $\begin{array}{l}\text { tell, say, famili, help, year, know, } \\
\text { want, parent, go, brother }\end{array}$ \\
\hline Sleep Issues & $\begin{array}{l}\text { sleep, feel, night, wake, head, voic, } \\
\text { hear, dream, think, thing }\end{array}$ \\
\hline Social/Life & $\begin{array}{l}\text { feel, want, friend, know, peopl, think, } \\
\text { talk, life, time, love }\end{array}$ \\
\hline Mental Health/PTSD & $\begin{array}{l}\text { feel, know, mental, time, think, year, } \\
\text { thing, help, happen, ptsd }\end{array}$ \\
\hline
\end{tabular}

Figure 4: Latent Dirichlet Allocation reveals prominent topics in mental health subreddits. A) Distribution of mid-pandemic posts from 15 mental health subreddits across 10 topics extracted using LDA on pre-pandemic mental health subreddit posts. Topic distribution was assessed for mid-pandemic posts between 2020-03-16 and 2020-04-20 to capture the phase of the pandemic right after stay-at-home orders had been announced or enacted for many areas in the United States. Inspection of the topic distribution indicated that there was minimal shift in most topics for all subreddits between the pre- and mid-pandemic timeframes (shown in Supplementary Figures S10 and S11). We tested changes in topic distributions across all 27 subreddits using a Wilcoxon signed-rank test (COVID19_support was not available during 2019). The prevalence of the Health Anxiety topic $(P=.008)$ and Life topic $(P=.014)$ significantly increased while the prevalence of the Alcohol/Addiction topic significantly decreased $(P=.004)$ between pre-pandemic to mid-pandemic timeframes. B) Manually labelled topics and top 10 terms associated with each topic derived from a LDA model created on mid-pandemic subreddit posts. 


\section{4. r/COVID19_support Characterization}

Figure 5 characterizes the new r/COVID19_support subreddit through supervised and unsupervised methods. Health Anxiety emerged as a major concern through classification (i.e., $\mathrm{r} /$ healthanxiety was classified most prevalently) and topic modelling while unsupervised clustering revealed a substantial portion of posts were assigned to the Suicidality cluster.

\section{A - Classification B - Topic Modeling C - Unsupervised Clustering}

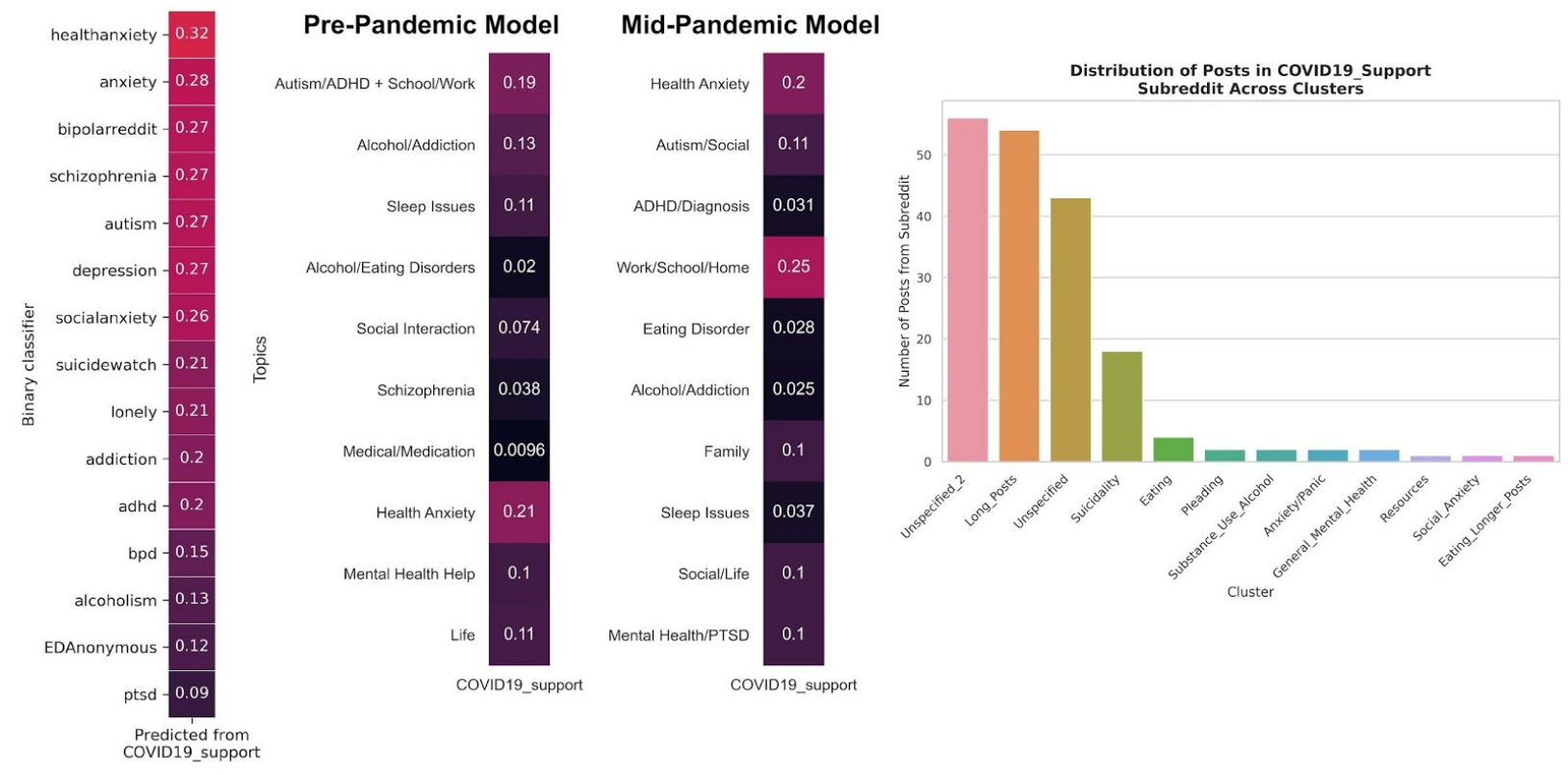

Figure 5: Characterization of r/COVID19_support through supervised and unsupervised methods. A) Proportion of r/COVID19_support posts (2020-03-11 to 2020-04-20) that each binary classifier trained on pre-pandemic data detects. B) Distribution of pre-pandemic model topics (left) and mid-pandemic model topics (right) for posts in r/COVID19_support, highlighting prominent topics in the posts such as health anxiety and issues in school, work, and home scenarios. The distribution of topics indicate common themes of pain points, which could help guide the medium and content of mental health resources. C) Distribution of unsupervised cluster representation among posts from r/COVID19_support. While many posts were assigned to unspecified clusters, the substantial portion of posts assigned to the Suicidality cluster is notable.

\subsection{Measuring Similarity Between Subreddits over Time with Supervised Dimensionality Reduction}

The highest silhouette score ( 0.93 ) was obtained with $\mathrm{n}$ neighbors $=200$ and $\min$ dist $=0.0$ and cosine distance. Using UMAP and true labels, we reduced feature sets for each post to 2D and measured the directed Hausdorff distances between the clusters in order to quantify which subreddits are converging or diverging as the pandemic advances (see Figure 6 and Supplementary Figure S12 for full results). 
A

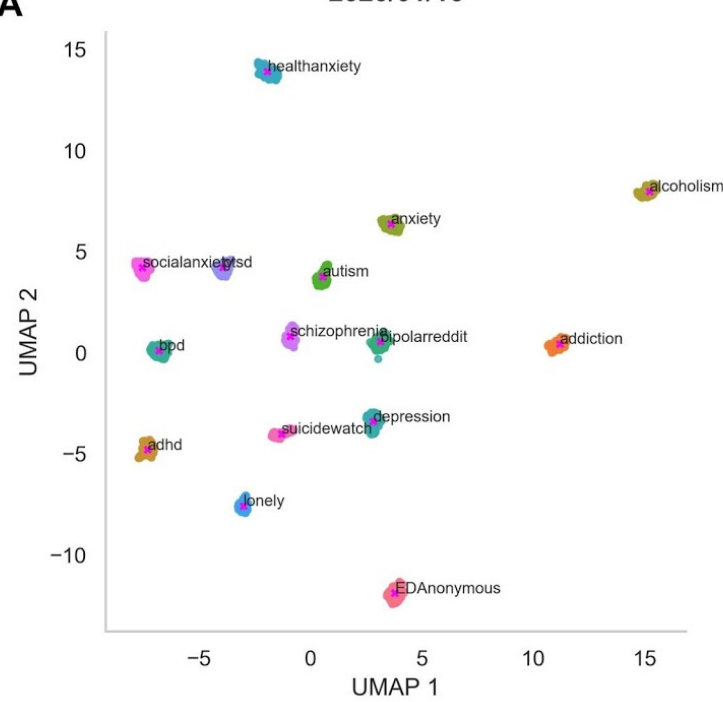

B

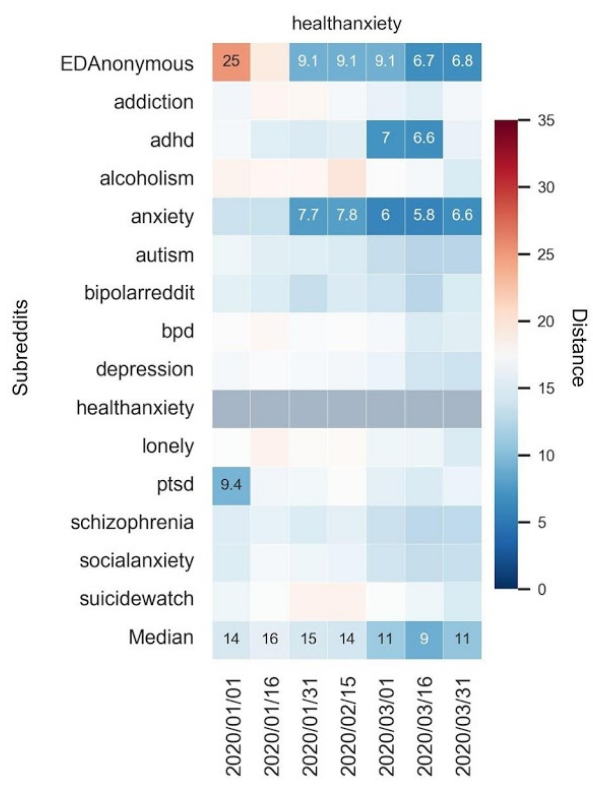

2020/03/01

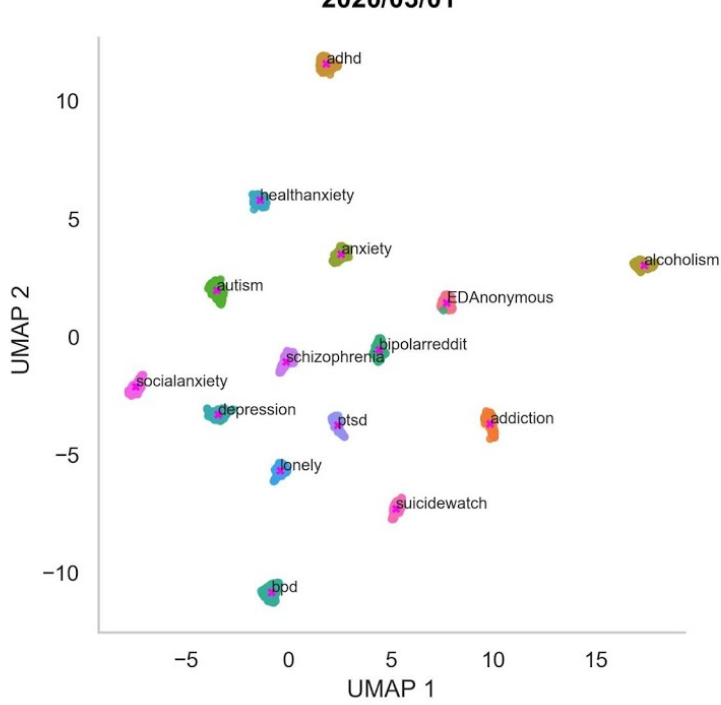

C

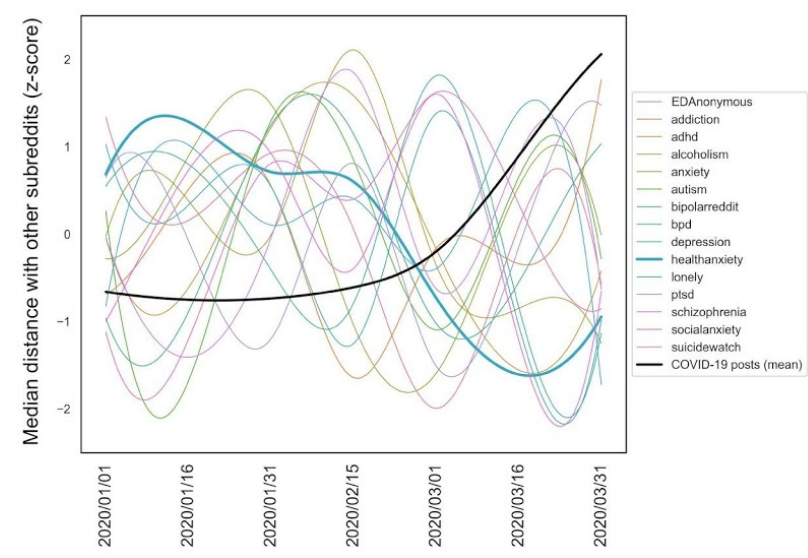

Figure 6. Supervised dimensionality reduction to measure how certain subreddits are becoming more or less similar over time. A) Supervised dimensionality reduction of posts within 15-day time windows with starting day displayed ( $\mathrm{r}$ /healthanxiety becomes more similar to other subreddits). B) Median pairwise distance with $\mathrm{r} /$ healthanxiety for each time window over 50 bootstrapping samples displaying only extreme values with regards to normal 2019 fluctuations (top and bottom 5th percentiles), which indicates they are less likely to be part of normal fluctuations in distance (see Supplementary Section 1.4 for a precision analysis of this approach and see Supplementary Figure S12 for full results). C) The median distance across all subreddits (last row in B) shows subreddits becoming more similar to $\mathrm{r}$ /healthanxiety during the increase in COVID-19 related posts (Figure 1 mean values were split into 7 time windows to match subreddit trends and the mean was taken for each window). $\mathrm{r} /$ healthanxiety is the only trend that significantly correlates with COVID-19 posts after Benjamini-Hochberg multiple comparison correction $(\rho=-0.96, P<.001)$. 


\section{Discussion}

We applied a broad array of natural language processing (NLP) techniques including statistical analysis of feature trends, supervised learning, interpretability, and unsupervised learning to measure how COVID-19 may have impacted different mental health support groups. We discuss each analysis in turn, focusing on clinical takeaways.

\subsection{Trend Analysis}

In our results, $r$ healthanxiety had several spikes in pandemic related posts in January before other subreddits were posting about a possible pandemic (see example of post in Table 2). This evidence supports concerns regarding the prolonged stress that people with health anxiety may be suffering. We then evaluated the nature of linguistic changes in mental health subreddits as the pandemic spread. Strikingly, many more linguistic features significantly changed from January to April of 2020 than in the same months of previous years (see Supplementary Fig. S5 for full results). The amount of posts significantly decreased in multiple subreddits in 2020, as tends to happen during that time period, but the decrease was often more than in previous years. This could be a concern if individuals are not using these subreddits to seek support and have limited access to other support structures. Interestingly, the amount of posts significantly grew for $\mathrm{r} /$ conspiracy, which is consistent with findings from other analyses [19]. A sign of concern comes from the increase in negative semantic features for certain subreddits, the highest of which belonged to the groups for ADHD, eating disorders, anxiety, and depression within the mental health subreddits and personal finance, relationships, and fitness within the non-mental health subreddits. Regarding ADHD, some parents in France of children and adolescents diagnosed with ADHD reported increased hyperactivity and inattention, while other parents reported symptomatic improvement [20]. There is also evidence that individuals with eating disorders are experiencing worse symptoms and heightened risk for relapse, hypothesized to stem from limited care access, less structure in daily activities, and decreased social support [21] and captured in the example in Table 2. We found an increase in the use of body-related words for $r$ anxiety and a decrease in $r$ /EDAnonymous and $r /$ fitness. Pandemic-related features (motion, isolation, economic stress, home) increased or decreased across some subreddits in the direction that would be expected for a pandemic and the amount of change provides further evidence of concerns or lack thereof. For instance, while the isolation feature significantly increased for most subreddits, it increased most for $\mathrm{r}$ /anxiety and did not increase for $\mathrm{r}$ /socialanxiety (i.e., users write less about isolation and loneliness; see r/socialanxiety example in Table 2).

\subsection{Classification and Feature Importance}

We successfully developed binary models to classify 2019 posts originating from a given mental health subreddit as distinct from posts originating from other mental-health related subreddits. We then used these models to classify $\mathrm{r} /$ COVID19 support posts and identify general distribution of complaints as well as identify posts potentially containing at-risk behavior (e.g., from $\mathrm{r} / \mathrm{SuicideWatch).} \mathrm{By} \mathrm{leveraging} \mathrm{interpretable} \mathrm{linear} \mathrm{methods,} \mathrm{we} \mathrm{were} \mathrm{able} \mathrm{to}$ establish features key to distinguishing mental health subreddits from one another (see Supplementary Tables S4 and S5 and the y-axis of Supplementary Fig. S5), which helps understand how different mental health concerns may manifest in language. Some of the most interesting top important features used to classify each subreddit were: gunning fog index (i.e., how readable the text is) for r/depression; the tokens 'cancer' and 'google' for $\mathrm{r} /$ healthanxiety; domestic stress and guns lexicons for r/ptsd; LIWC 'religion' and number of long words for schizophrenia; LIWC see (e.g., 'picture', 'screen', 'stare') for r/socialanxiety; LIWC money and certainty for $\mathrm{r} /$ SuicideWatch (see Table 2 for more examples).

\subsection{Unsupervised Methods: Topic Modeling and Clustering}

We established important conversation topics that span across mental health support subreddits through unsupervised methods (LDA and clustering). Unsupervised clusters such as Seeking Advice, Resources, Seeking Normalization, Suicidality, and Medication varied in representation across the mental health subreddits and provided 
insight into the forms of discussion occurring. This cluster structure has utility for assessing changes in discussion on mental health and for analyzing subreddits like r/COVID19_support, on which many posts were found to map to the Suicidality cluster. r/bpd (Borderline Personality Disorder) and r/ptsd (Post-Traumatic Stress Disorder) became significantly enriched for posts from the Suicidality cluster in the mid-pandemic dataset. The r/anxiety subreddit became significantly enriched for posts from the health anxiety cluster, which captures the general theme of heightened health anxiety. Topic modeling found an increase in the distribution of the Health Anxiety topic across mid-pandemic posts, and also highlighted changes in topics between pre- and mid-pandemic timeframes, such as the introduction of the topics Social Interaction and Mental Health Help. Additionally, a large number of posts in the r/COVID19_support group were identified to relate to the Health Anxiety topic and to voice concerns with daily living at home, school, and work. Posts in these topics and clusters could be important for subreddit moderators to track as they seek to cultivate a culture of support and provide effective assistance to authors in crisis (e.g., tracking clusters like Seeking Advice, Medication, Suicidality, and Resources) and as they seek to understand the chief concerns of their communities (e.g, tracking topics like sleep issues and social interaction).

\subsection{Measuring Similarity Between Subreddits over Time with Supervised Dimensionality Reduction}

Psychiatric care for patients during the pandemic should be informed by an understanding of possible convergence among some disorders, which could merit a blending of standard treatment approaches, and of possible separation of certain clusters from the rest, which could identify an at-risk population. Overall, the more users were posting about COVID-19, the more similar subreddits became to $\mathrm{r}$ /healthanxiety (see Figure 6C). The $\mathrm{r}$ /healthanxiety similarity to other subreddits was the only subreddit that significantly correlated with the rise in COVID-19 related posts. Interestingly, the subreddits that became most negative per trend analyses, $r$ /ADHD, $r$ /EDAnonymous and $r / A n x i e t y$, also became most like r/healthanxiety during the general spike of COVID-19 related posts during March 2020. These results suggest a clinically-testable hypothesis that the symptom of health anxiety may have increased most in the psychiatric populations corresponding to these three subreddits.

\subsection{Limitations}

Our study population is not characterized with formally-documented clinical diagnoses, although some post authors make diagnostic claims. For example, 5\% posts in our full 2019 dataset are authored by individuals who make a diagnostic claim (e.g., "I have obsessive compulsive disorder"). We suspect the prevalence of mental health disorders among post authors on these fora is much higher, given the psychiatric experiences described in these posts. If further application of NLP techniques can expand the set of post authors with high likelihood of clinical diagnoses, analyses could be restricted to that subset of authors without loss of sufficient post volume. Furthermore, while linguistic changes occurred during the pandemic, in this study we do not causally link any individual changes to specific events.

\subsection{Future Directions}

Extremely risky behavior is common content in these posts including asking for advice for restricting food in $\mathrm{r}$ /EDAnonymous or planning suicide in $\mathrm{r} /$ SuicideWatch; therefore, more clinical attention is urgently needed to provide effective resources to Reddit users. Furthermore, understanding the nature of posts from subreddits other than $\mathrm{r} /$ SuicideWatch (including $\mathrm{r} / \mathrm{COVID} 19$ support) that were classified as $\mathrm{r} / \mathrm{SuicideWatch}$ posts or that belong to the Suicidality cluster demands further research. Critically, these posts were made on subreddits without the policies and response systems used by moderators on $\mathrm{r} /$ SuicideWatch itself and whose authors may benefit from urgent intervention. NLP applied to Reddit posts could help direct users to tailored resources or more ideal subreddits where it is more likely to receive support and help with the triage of moderator responses. For example authors of posts in the Seeking Normalization cluster may be looking for solidarity whereas authors of posts with high usage of the Sleep Issues LDA topic may benefit from sleep-related advice. These analyses of Reddit could also be followed by related analyses of posts on Twitter, which enables some geolocation but restricts anonymity and post length. Geographic information could, for example, be used to characterize the impact on mental health of specific policy 
developments like the imposition of state-wide lockdowns that varied in time across geographic areas. Taking trend analysis and supervised dimensionality reduction, one can track subreddits that did not change and more research is needed to understand if certain groups were more resilient to the pandemic. Ultimately, we have found many linguistic patterns for specific mental health groups, and these patterns could be studied further in clinical settings that include formal diagnoses and more extensive covariate information (e.g. racial or socioeconomic background).

\subsection{Conclusions}

We performed successful classification among mental health subreddits and identified important features to understand how each mental health problem may manifest in language. We found a very early spike in COVID-19 related posts in $\mathrm{r}$ /healthanxiety in January 2020, which would be interesting to replicate if new epidemics appear. We tracked features across time and observed the largest negative semantic changes during the COVID-19 pandemic for $\mathrm{r} / \mathrm{ADHD}, \mathrm{r} /$ EDAnonymous (eating disorders) and $\mathrm{r} /$ Anxiety, which were the same groups that become most similar to $\mathrm{r}$ /healthanxiety during the rise of COVID-19 posts in March. Understanding linguistic features that distinguish these communities and how language use has changed during the pandemic has generated several important hypotheses for evaluation in clinical settings that may help inform the provision of responsive care. Our findings suggest the pandemic may have induced health anxiety among several mental health and non-mental health communities given that a) posts on r/COVID19_support were classified most frequently as belonging to $\mathrm{r} /$ healthanxiety, b) mid-pandemic posts from the r/anxiety subreddit became significantly enriched for posts from the Health Anxiety cluster, c) LDA topic analysis found the Health Anxiety topic significantly increased in the mid-pandemic posts compared to the pre-pandemic posts, and d) supervised dimensionality reduction revealed that the more users posted about COVID-19 the more similar subreddits became to $r$ /healthanxiety. Suicidality was another concerning theme given that unsupervised clustering revealed the Suicidality cluster doubled in size and a new cluster surrounding self-harm emerged. Notably, a substantial portion of posts from r/COVID19_support were assigned to the Suicidality cluster, and two subreddits ( $\mathrm{r} / \mathrm{bpd}$ and $\mathrm{r} / \mathrm{ptsd}$ ) became significantly associated with the Suicidality cluster during the pandemic. Furthermore, 26\% of posts from COVID19_support were classified by $\mathrm{r} /$ SuicideWatch by its binary classifier. We hope this work will bring attention to these large online communities' needs and concerns, since Reddit may be the first path to treatment for many online users and these subreddits currently lack sufficient resources. We have helped to define some of these concerns through multiple methods such as feature importance (e.g., the guns lexicon is important for classifying $\mathrm{r} / \mathrm{ptsd}$ ), trend analysis (e.g., decreased use over time of the motion lexicon in r/anxiety), unsupervised clustering (e.g., a substantial number of posts on COVID19_support mapped to a Suicidality post cluster and subreddits show discussion of topics distinct from the overall subreddit theme), topic modeling (e.g., the high rate of concerns regarding home, work, and school on r/COVID19_support). We further hope that insights from this work will deepen our understanding of mental health challenges during the pandemic, inform the provisioning of appropriate therapeutic resources, and inspire greater use of NLP for the inspection of world-changing events such as epidemics, elections, and protests.

Data Availability Statement: All data and code are available through the Open Science Framework (https://osf.io/7peyq/) and https://github.com/danielmlow/reddit.

Acknowledgements: We thank the course staff of MIT's Machine Learning for Healthcare class, who provided feedback on the project structure and analysis and Kate H. Bentley and Mostafa Abdou who provided feedback on the manuscript.

Funding: D.M.L. was supported by the NIH-NIDCD T32 (5T32DC000038-28). T.T. was supported by the NIH-NIDCD T32 (DC000038). L.R. was supported by the NIH-NHGRI T32 (5T32HG002295-12). The work was supported by a gift to the McGovern Institute for Brain Research at MIT.

Author Contribution Statement: D.L., L.R., and T.T. conceived the experimental design, performed data analysis, and drafted the manuscript. All authors provided feedback on analyses and reviewed the manuscript.

Competing Interests: J.T. reports unrelated research support from Otsuka. None of the authors declare any competing interests. 
Distribution Statement: Approved for public release. Distribution is unlimited. This material is based upon work supported by the Under Secretary of Defense for Research and Engineering under Air Force Contract No. FA8702-15-D-0001. Any opinions, findings, conclusions or recommendations expressed in this material are those of the author(s) and do not necessarily reflect the views of the Under Secretary of Defense for Research and Engineering. (C) 2020 Massachusetts Institute of Technology. Delivered to the U.S. Government with Unlimited Rights, as defined in DFARS Part 252.227-7013 or 7014 (Feb 2014). Notwithstanding any copyright notice, U.S. Government rights in this work are defined by DFARS 252.227-7013 or DFARS 252.227-7014 as detailed above. Use of this work other than as specifically authorized by the U.S. Government may violate any copyrights that exist in this work.

\section{References}

1. Brooks SK, Webster RK, Smith LE, Woodland L, Wessely S, Greenberg N, Rubin GJ. The psychological impact of quarantine and how to reduce it: rapid review of the evidence. Lancet 2020; [doi: $10.1016 / \mathrm{S} 0140-6736(20) 30460-8]$

2. Duan L, Zhu G. Psychological interventions for people affected by the COVID-19 epidemic. Lancet Psychiatry. thelancet.com; 2020. p. 300-302. PMID:32085840

3. Alonzi S, La Torre A, Silverstein MW. The psychological impact of preexisting mental and physical health conditions during the COVID-19 pandemic. Psychological Trauma : Theory, Research, Practice and Policy 2020 Aug 12;S1:S236-S238. PMID:32525380

4. Wardell J, Kempe T, Rapinda KK, Single AN, Bilevicius E, Frohlich JR, Hendershot CS, Keough MT. Drinking to cope during the COVID-19 pandemic: The role of external and internal stress-related factors in coping motive pathways to alcohol use, solitary drinking, and alcohol problems. PsyArXiv. 2020. [doi: $10.31234 /$ osf.io/8vfp9]

5. Guntuku SC, Yaden DB, Kern ML, Ungar LH, Eichstaedt JC. Detecting depression and mental illness on social media: an integrative review. Current Opinion in Behavioral Sciences. 2017. p. 43-49. [doi:

10.1016/j.cobeha.2017.07.005]

6. Calvo RA, Milne DN, Sazzad Hussain M, Christensen H. Natural language processing in mental health applications using non-clinical texts. Natural Language Engineering. 2017. p. 649-685. [doi: $10.1017 / \mathrm{s} 1351324916000383]$

7. Shen JH, Rudzicz F. Detecting anxiety through reddit. Proceedings of the Fourth Workshop on Computational Linguistics and Clinical Psychology — From Linguistic Signal to Clinical Reality Association for Computational Linguistics; 2017. p. 58-65.

8. Sekulić I, Gjurković M, Šnajder J. Not Just Depressed: Bipolar Disorder Prediction on Reddit. Proceedings of the 9th Workshop on Computational Approaches to Subjectivity, Sentiment and Social Media Analysis Association for Computational Linguistics; 2018. [doi: 10.18653/v1/W18-6211]

9. Yates A, Cohan A, Goharian N. Depression and Self-Harm Risk Assessment in Online Forums. Proceedings of the 2017 Conference on Empirical Methods in Natural Language Processing [Internet] Association for Computational Linguistics; 2017. [doi: 10.18653/v1/D17-1322]

10. Zirikly A, Resnik P, Uzuner O, Hollingshead K. CLPsych 2019 shared task: Predicting the degree of suicide 
risk in Reddit posts. Proceedings of the Sixth Workshop on Computational Linguistics and Clinical Psychology Association for Computational Linguistics; 2019. p. 24-33.

11. Pennebaker JW, Booth RJ, Boyd RL, Francis ME. LIWC 2015 Operator's Manual. Austin, TX: Pennebaker Conglomerates Inc; 2015.

12. De Choudhury M, Gamon M, Counts S, Horvitz E. Predicting depression via social media. Proceedings of the Seventh International AAAI Conference on Weblogs and Social Media AAAI Publications; 2013.

13. Park A, Conway M, Chen AT. Examining Thematic Similarity, Difference, and Membership in Three Online Mental Health Communities from Reddit: A Text Mining and Visualization Approach. Computers in Human Behavior Elsevier; 2018 Jan;78:98-112. PMID:29456286

14. Park A, Conway M. Tracking Health Related Discussions on Reddit for Public Health Applications. AMIA Annu Symp Proc 2018;2017:1362-1371. PMID:29854205

15. Reddit.com desktop traffic share 2020. Statista. [cited 2020 Jun 19]. Available from: https://www.statista.com/statistics/325144/reddit-global-active-user-distribution/

16. Barthel M, Stocking G, Holcomb J, Mitchell A. Reddit news users more likely to be male, young and digital in their news preferences. Pew Research Center [Internet] 2016; Available from: https://www.pewresearch.org/wp-content/uploads/sites/8/2016/02/PJ_2016.02.25_Reddit_FINAL.pdf

17. Baumgartner JM. Pushshift API. 2018. Available from: pushshift.io

18. McInnes L, Healy J, Melville J. UMAP: Uniform Manifold Approximation and Projection for Dimension Reduction. arXiv [statML]. 2018. Available from: http://arxiv.org/abs/1802.03426

19. Shahsavari S, Holur P, Tangherlini TR, Roychowdhury V. Conspiracy in the Time of Corona: Automatic detection of Covid-19 Conspiracy Theories in Social Media and the News. arXiv [csCL]. 2020. Available from: http://arxiv.org/abs/2004.13783

20. Bobo E, Lin L, Acquaviva E, Caci H, Franc N, Gamon L, Picot M-C, Pupier F, Speranza M, Falissard B, Purper-Ouakil D. How do children and adolescents with Attention Deficit Hyperactivity Disorder (ADHD) experience lockdown during the COVID-19 outbreak? Encephale 2020 Jun 7;46(3S):S85-S92.

PMID:32522407

21. Termorshuizen JD, Watson HJ, Thornton LM, Borg S, Flatt RE, MacDermod CM, Harper LE, van Furth EF, Peat CM, Bulik CM. Early Impact of COVID-19 on Individuals with Eating Disorders: A survey of 1000 Individuals in the United States and the Netherlands. medRxiv. 2020. [doi: 10.1101/2020.05.28.20116301] 


\section{Supplementary Materials}

\section{Methods}

\subsection{Data downloading and preprocessing}

Posts were extracted from the 28 subreddits listed in the main body, including 15 mental health subreddits, 11 non-mental health subreddits, and the broad mental health subreddits, $r$ /mentalhealth and r/COVID19_support. Only original posts and not comments were included. Only posts including a title and a body were included to exclude posts with just images. Titles and bodies of posts were concatenated. Posts were filtered to only be in English. We only kept the first post from each user in each timeframe and to remove posts from bots and ads. Posts from the following timeframes were downloaded:

- Mid-pandemic data (January to April 2020): all posts on each subreddit were extracted between the dates of 2020-01-01 and 2020-04-20. Unique users: 320,364.

- Pre-pandemic data: all posts made between 2018-11-01 and 2019-11-01 were extracted. Unique users: 327,289 .

Posts were either randomly downsampled to 30,000 per subreddit, or all included, with a maximum of 1000 posts extracted per day following pushshift.io limitations. Classification, unsupervised clustering and topic modeling used pre-pandemic data and mid-pandemic data. Supplement Figure 1 in provides histograms of how many posts were extracted from each subreddit for the time-frames used in analysis.

A

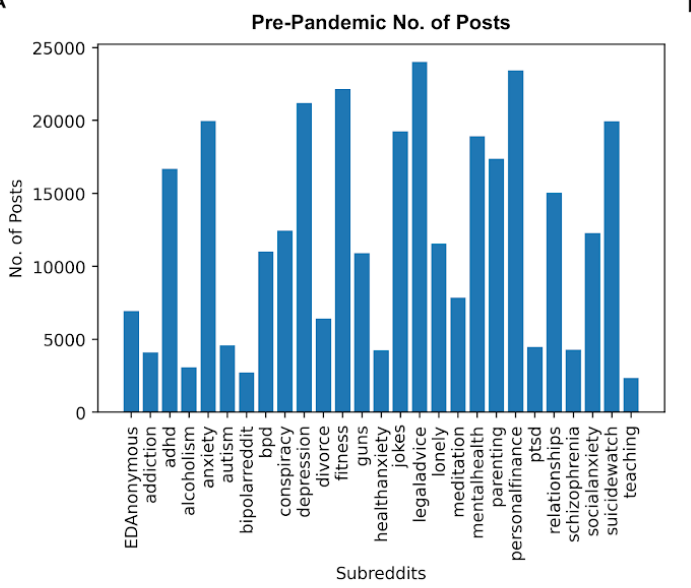

B

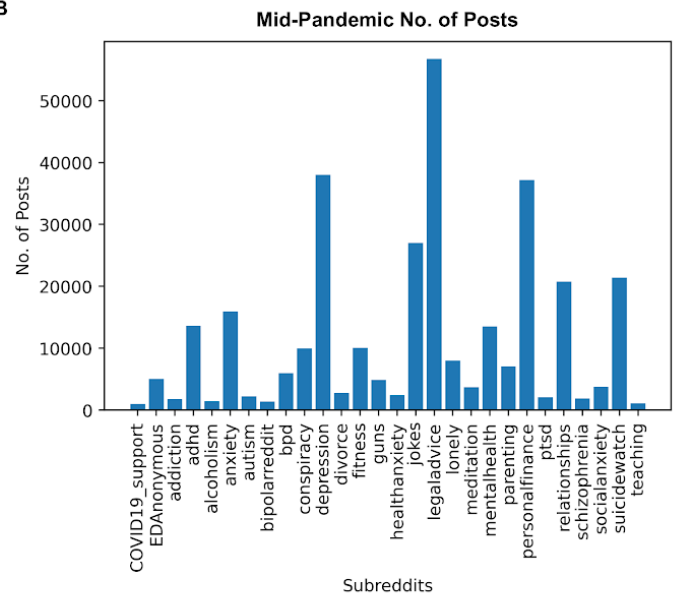

Figure S1: Distribution of extracted posts over all subreddits. The number of posts extracted from each subreddit in our data set for A) pre-pandemic and B) mid-pandemic timeframes.

The trend analysis used mid-pandemic data and the 2019 and 2018 additional control datasets:

- 2019 control for mid-pandemic (January to April 2020): all posts made between 2019-01-01 and 2019-04-20 were extracted. Unique users: 282,560.

- 2018 control for mid-pandemic (January to April 2020): all posts made between 2018-01-01 and 2018-04-20 were extracted. Unique users: 177,089.

Unique users across all time windows (pre and 2019 overlap): 826,961. 


\subsection{Feature extraction}

The following features were extracted from each post (resulting dimensions): VADER sentiment analysis (3) [1]; Textacy basic word and syllable counts (8) [2]; Textacy readability metrics (9); Linguistic Inquiry and Word Count, which includes words scored by their belonging to different semantic and grammatical categories such as 'body' or 'pronouns' (62) [3]; TF-IDF unigrams and bigrams (256-1024 depending on analysis; see below); punctuation count (1); and the following manually built lexicons: suicidality (1); economic stress (1); isolation (1); substance use (1); domestic stress (1); guns (1) (see Table S1 for tokens that make up the lexicons). Lexicon counts were normalized by the amount of words in each post. For LIWC extraction and TF-IDF calculation, all words were lemmatized and stemmed using the NTLK package. Furthermore for TF-IDF calculation, English stopwords and words with three or fewer characters were removed from posts. Further preprocessing depended on the analysis:

Classification: all features were used but TF-IDF (256 features) was fit on the train set and transformed on the test set separately to avoid overfitting.

Trend Analysis: all features except TF-IDF were used for Figures 2, S5, S6, S7. An additional feature quantifying the presence of tokens from a COVID-19 lexicon (see Table S1) was computed for Figures 1 and S3.

LDA: After utilizing the same pre-processing steps as TF-IDF, a dictionary was created with the top 100,000 unigrams and bigrams from all of the posts such that each dictionary token appeared in more than $0.1 \%$ of the training posts but less than $50 \%$ of the posts.

Unsupervised clustering: Features used for unsupervised clustering included the top 1024 TF-IDF ngrams as well as the non-TF-IDF features listed above.

Supervised dimensionality reduction: we used all features including 256 features for TF-IDF.

\begin{tabular}{|c|c|}
\hline Theme & Terms \\
\hline Economic Stress & $\begin{array}{l}\text { Unemploy, economy, rent, mortgage, evict, enough money, more money, pay the bills, owe, } \\
\text { debt, make ends meet, afford, save enough, salary, wage, income, job, eviction }\end{array}$ \\
\hline Isolation & $\begin{array}{l}\text { alone, lonely, no one cares about me, no one cares, can't see anyone, can't see my, i miss } \\
\text { my, i want to see my, trapped, i'm in a cage, lonely, feel ignored, ignoring me, ugly, } \\
\text { rejected, avoid, avoiding me, am single, been single, quarantine, lockdown, isolation, } \\
\text { self-isolation }\end{array}$ \\
\hline Substance Use & $\begin{array}{l}\text { smoke, cigarette, tobacco, wine, drink, beer, alcohol, drug, opioid, cocaine, snort, vodka, } \\
\text { whiskey, whisky, tequila, meth }\end{array}$ \\
\hline Guns & gun, pistol, revolver, semiautomatic, rifle, shoot, firearm, semi-automatic \\
\hline Domestic Stress & $\begin{array}{l}\text { divorce, domestic violence, abuse, yelling, fighting with me, we're fighting, single mom, } \\
\text { single dad, single parent, hit me, slapped me, fighting, fight }\end{array}$ \\
\hline Suicidality & $\begin{array}{l}\text { commit suicide, jump off a bridge, i want to overdose, i'm a burden, i'm such a burden, i } \\
\text { will overdose, thinking about overdose, kill myself, killing myself, hang myself, hanging } \\
\text { myself, cut myself, cutting myself, hurt myself, hurting myself, want to die, wanna die, } \\
\text { don't want to wake up, don't wake up, never want to wake up, don't want to be alive, want } \\
\text { to be alive, wish it would all end, done with living, want it to end, it all ends tonight, live } \\
\text { anymore, living anymore, life anymore, be dead, take it anymore, end my life, think about } \\
\text { death, hopeless, hurt myself, no one will miss me, don't want to wake up, if i live or die, i }\end{array}$ \\
\hline
\end{tabular}




\begin{tabular}{|l|l|}
\hline & hate my life, shoot me, kill me, suicide, no point \\
\hline COVID-19 & $\begin{array}{l}\text { corona, virus, viral, covid, sars, influenza, pandemic, epidemic, quarantine, lockdown, } \\
\text { distancing, national emergency, flatten, infect, ventilator, mask, symptomatic, epidemiolog, } \\
\text { immun, incubation, transmission, vaccine }\end{array}$ \\
\hline
\end{tabular}

Table S1: Manually constructed lexicons. Developed to assess the prevalence of tokens related to these topics in all of the subreddits.

\subsection{Latent Dirichlet Allocation Topic Modeling on Pre- and Mid-Pandemic Subreddits}

The dictionary created as described in Section 1.2 was applied to all posts to create a bag- of-words corpus which was used to create an LDA model using 25 passes and 3 workers. Models with 5, 10, 15, 20, and 25 topics were created. Models were also generated multiple times with different subsamples of posts to assess stability of topics. A manually chosen LDA model with 10 topics was then applied to all posts across all subreddits (mental health and non-mental health) to assess the distribution of topics, allowing for comparison between the distribution of posts pre-pandemic vs mid-pandemic. A manually chosen LDA model created on mid-pandemic data was applied to posts from r/COVID19_support to assess any change in topic distribution.

\subsection{Measuring Similarity Between Subreddits over Time with Supervised Dimensionality Reduction}

Since UMAP contains parameters that could affect relative distance between subreddits as could downsampling the data to obtain balanced classes, we estimated the precision of this approach on 2019 data. First, hyperparameter tuning was performed (2700 samples for each subreddit) to find the parameter set that optimized clustering measured through silhouette score using $\mathrm{n}$ neighbors $(2,10,20,50,100,200)$, min dist $(0.0,0.1,0.25,0.5,0.8,0.99)$ and metric (euclidean, cosine). Second, to tackle the variance caused by subsampling, we measured the pairwise Hausdorff distances between 2019 clusters across 50 runs, each with new random subsampling. Using a distance metric between clusters, rather than their absolute centroid location, allows for avoiding rotation or flipping effects of dimensions. Bootstrapping across runs provides an estimate of the method's precision and also allows us to measure how rare 2020 changes in distances are with respect to a distribution of regular fluctuations for a non-pandemic year (2019). For 2020, we also compute the median distance across 50 bootstrapping samples for our final analysis. 


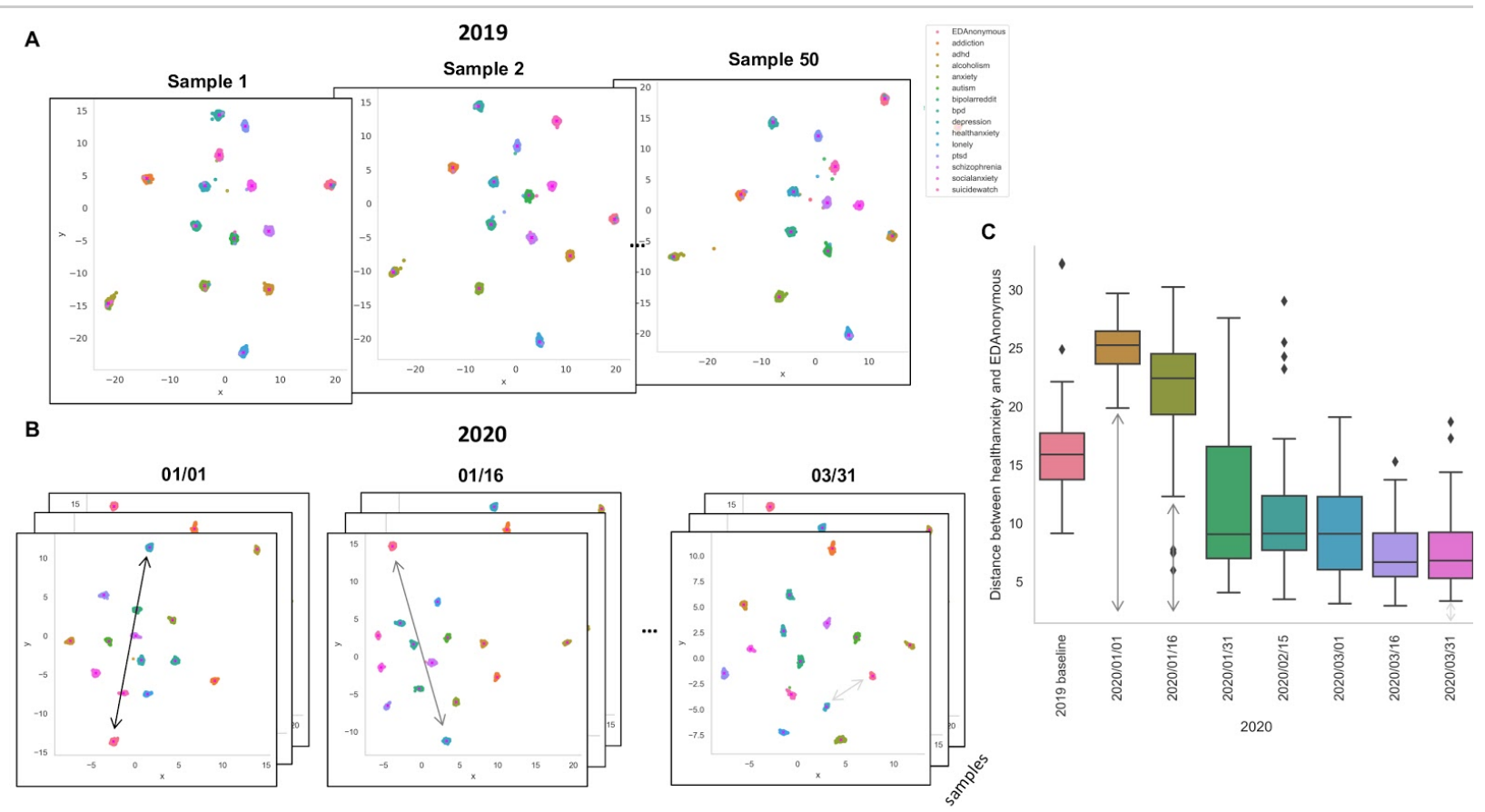

Figure S2. Procedure to establish precision of supervised UMAP for measuring distances between subreddits.

A) Normal fluctuations in pairwise distances for a given year when subsampling different random samples resulting in the 2019 distribution as observed in C. B) Distances when sampling 15-day time windows during 2020. As an example, arrows represent the distance between $\mathrm{r}$ /healthanxiety and $\mathrm{r}$ /EDAnonymous. C) The distributions built in A and B along with arrows represented in B. This allows us to show which changes in 2020 distances are not likely due to normal fluctuations in the data. 


\section{Results}

\subsection{Classification and Interpretability}

\begin{tabular}{|c|c|c|c|c|c|}
\hline Subreddit & SGD L1 & SGD EN & SVM & $\mathbf{E T}$ & XGB \\
\hline EDAnonymous & 0.898 & 0.898 & 0.904 & 0.848 & 0.887 \\
\hline addiction & 0.894 & 0.877 & 0.890 & 0.827 & 0.885 \\
\hline adhd & 0.849 & 0.855 & 0.860 & 0.791 & 0.831 \\
\hline alcoholism & 0.926 & 0.929 & 0.936 & 0.904 & 0.926 \\
\hline anxiety & 0.794 & 0.704 & 0.812 & 0.748 & 0.805 \\
\hline autism & 0.874 & 0.862 & 0.879 & 0.811 & 0.851 \\
\hline BipolarReddit & 0.811 & 0.789 & 0.811 & 0.746 & 0.787 \\
\hline bpd & 0.795 & 0.784 & 0.801 & 0.740 & 0.777 \\
\hline depression & 0.801 & 0.784 & 0.795 & 0.709 & 0.749 \\
\hline healthanxiety & 0.891 & 0.898 & 0.906 & 0.860 & 0.884 \\
\hline lonely & 0.859 & 0.864 & 0.863 & 0.824 & 0.850 \\
\hline ptsd & 0.895 & 0.887 & 0.889 & 0.850 & 0.883 \\
\hline schizophrenia & 0.828 & 0.811 & 0.827 & 0.762 & 0.800 \\
\hline socialanxiety & 0.827 & 0.839 & 0.841 & 0.775 & 0.833 \\
\hline SuicideWatch & 0.830 & 0.812 & 0.846 & 0.780 & 0.800 \\
\hline Mean & 0.851 & 0.840 & 0.857 & 0.798 & 0.837 \\
\hline Model complexity No. (\%) & $2503(48)$ & $3332(64)$ & $4316(83)$ & $5190(100)$ & $5190(100)$ \\
\hline
\end{tabular}

Table S2. Weighted F1 of independent binary classifiers. Model complexity is defined as the amount of non-zero features of the total 5190 features across classifiers $(15 \times 346)$. SGD L1: Stochastic gradient descent linear classifier with L1 penalty; SGD EN: Stochastic gradient descent linear classifier with elastic net penalty; SVM: linear support vector machine; ET: extra trees classifier; XGB: extreme gradient boosting classifier. 


\begin{tabular}{|c|c|c|c|}
\hline Subreddit & F1 pre-pandemic & F1 mid-pandemic & Change \\
\hline autism & 0.874 & 0.800 & 0.074 \\
\hline BipolarReddit & 0.811 & 0.753 & 0.058 \\
\hline healthanxiety & 0.891 & 0.834 & 0.057 \\
\hline depression & 0.801 & 0.761 & 0.04 \\
\hline anxiety & 0.794 & 0.755 & 0.039 \\
\hline addiction & 0.894 & 0.867 & 0.027 \\
\hline ptsd & 0.895 & 0.869 & 0.026 \\
\hline alcoholism & 0.926 & 0.903 & 0.023 \\
\hline socialanxiety & 0.827 & 0.836 & 0.009 \\
\hline SuicideWatch & 0.830 & 0.837 & 0.007 \\
\hline EDAnonymous & 0.898 & 0.891 & 0.007 \\
\hline bpd & 0.795 & 0.801 & 0.006 \\
\hline lonely & 0.859 & 0.864 & 0.005 \\
\hline adhd & 0.849 & 0.852 & 0.003 \\
\hline schizophrenia & 0.828 & 0.829 & 0.001 \\
\hline Mean & 0.851 & 0.83 & 0.025 \\
\hline
\end{tabular}

Table S3. Comparing performance of pre-pandemic baseline model on pre- and mid- pandemic test sets. Larger differences may indicate dataset shift during the spread of COVID-19. 


\subsection{Trend Analysis}

See Figure S4 for examples of trends and regression. See Figure S5 for 2020 main results and Figures S6 and S7 for comparisons to 2019 and 2018 trends. See Table 2 in the main text for examples of significant trends in Figure S5.

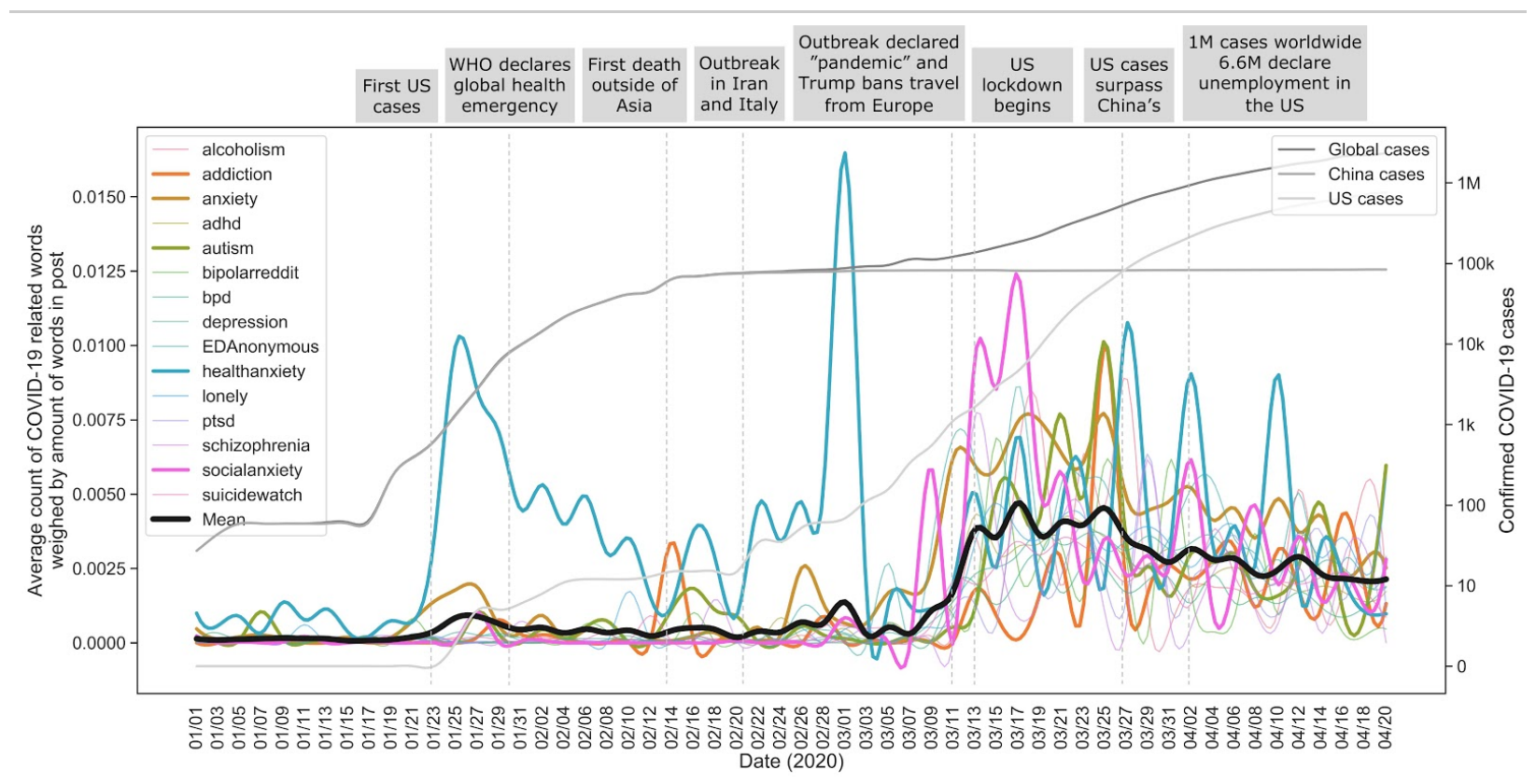

Figure S3. Amount of posts weighed by how much they are about COVID-19.

Timeline landmarks are chosen from NBC News timeline. The acute rise in COVID19 related posts occurs around March 11. The correlation between mean proportion of COVID-19 related posts and global COVID-19 cases is $\rho=$ $0.82(P<0.001)$. The health anxiety subreddit has a large increase in COVID-19 related posts almost two months before the general increase. The social anxiety subreddit has an increase during the beginning of the lockdowns in the US. 

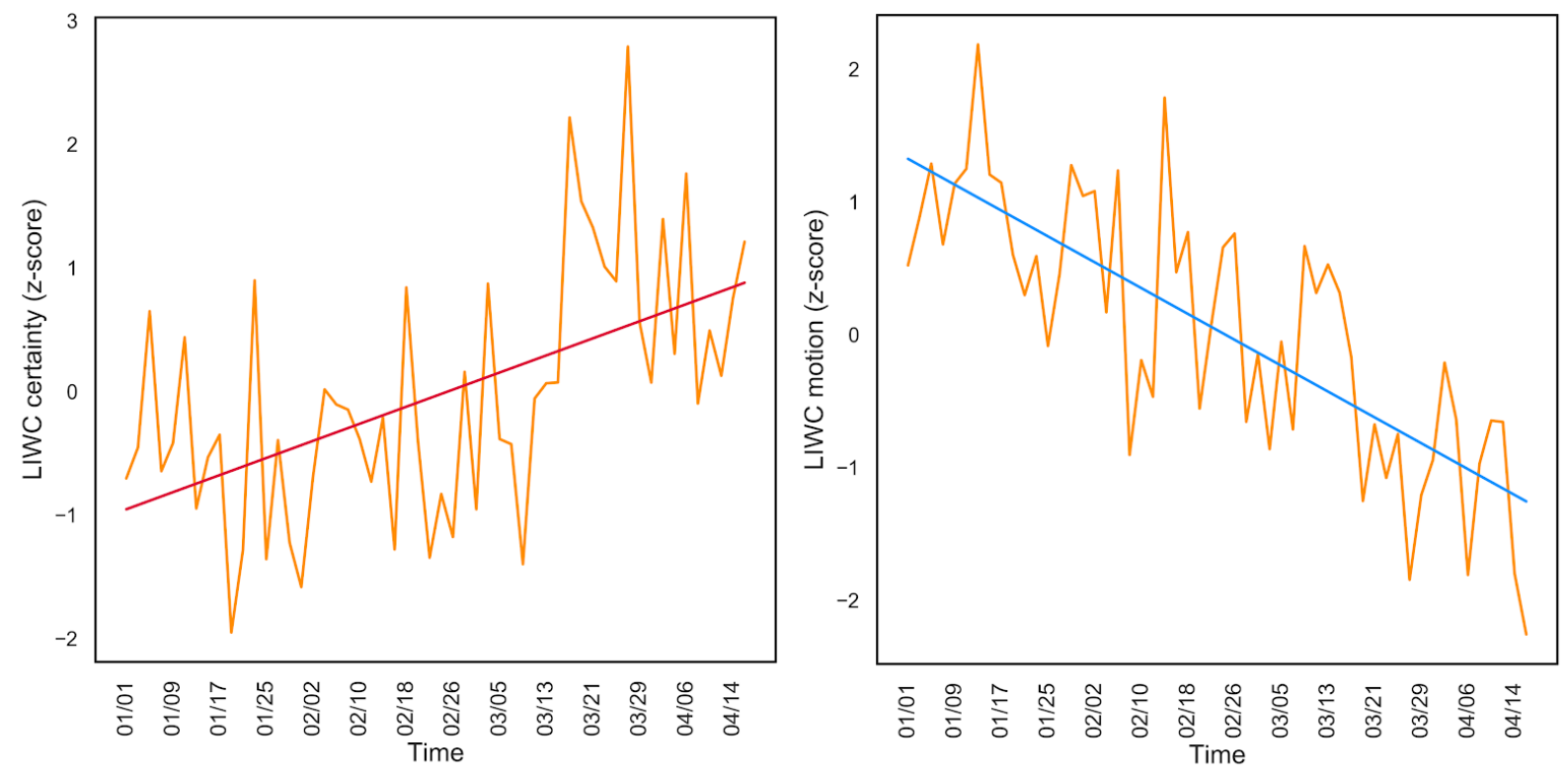

Figure S4. Examples of trends and regression for a single feature and subreddit. Example from r/anxiety of trends and color code for Figure 2 and Figures S5, S6, and S7. The mean value for each time window is computed and then a regression is fit. Positive slopes are in red and negative slopes and in blue. 


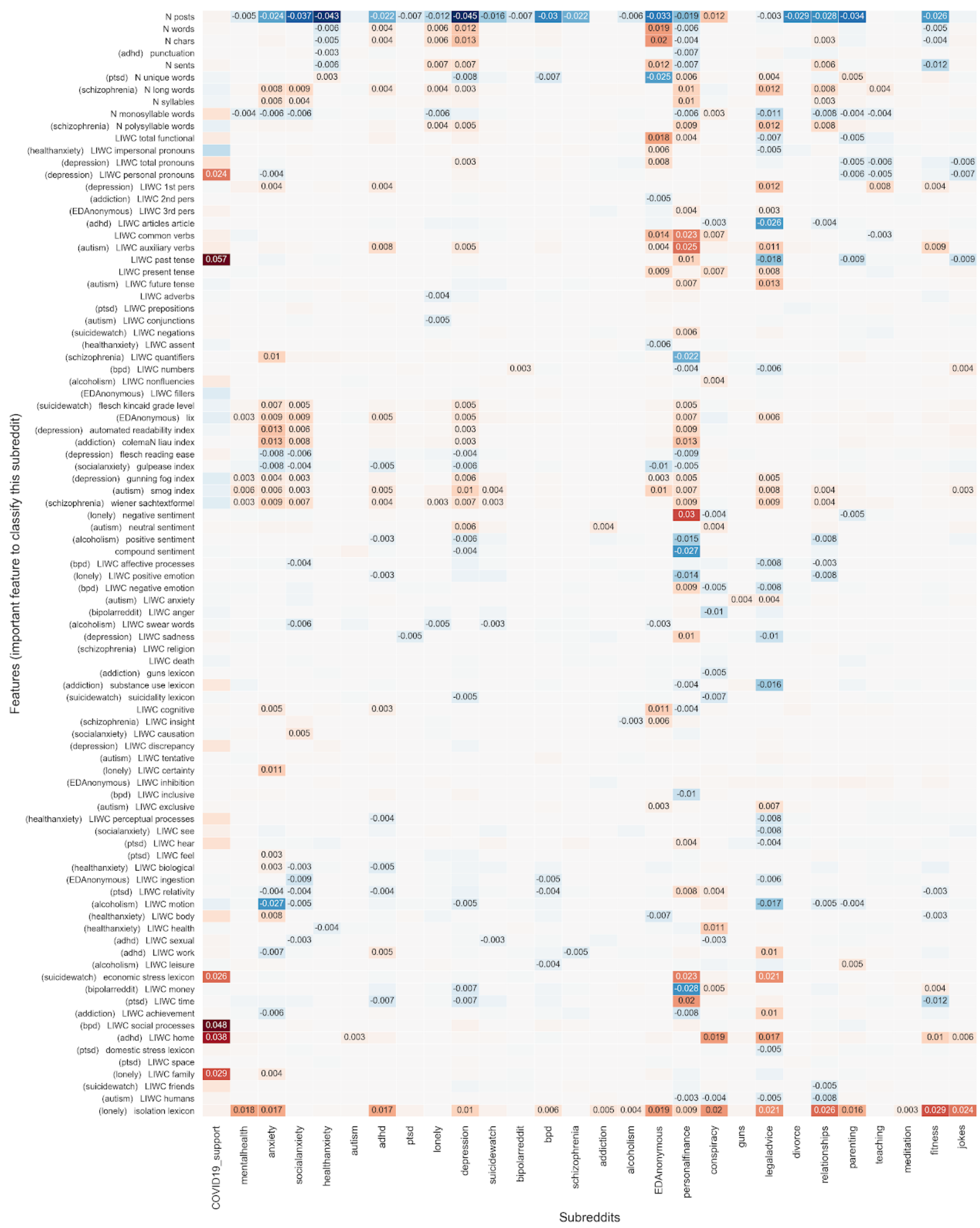

Figure S5. Full results for $\mathbf{2 0 2 0}$ trend analysis. Trends of mean feature values across subreddits from 2020-03-11 to 2020-03-20. We concatenated for which binary classifier they have the highest coefficient and thus are important to classify (only using upper half of coefficient values, which is why not all features are linked to a subreddit). Benjamini-Hochberg procedure was applied for multiple comparison (uncorrected alpha $=0.05$ ) and non-significant results are masked. 


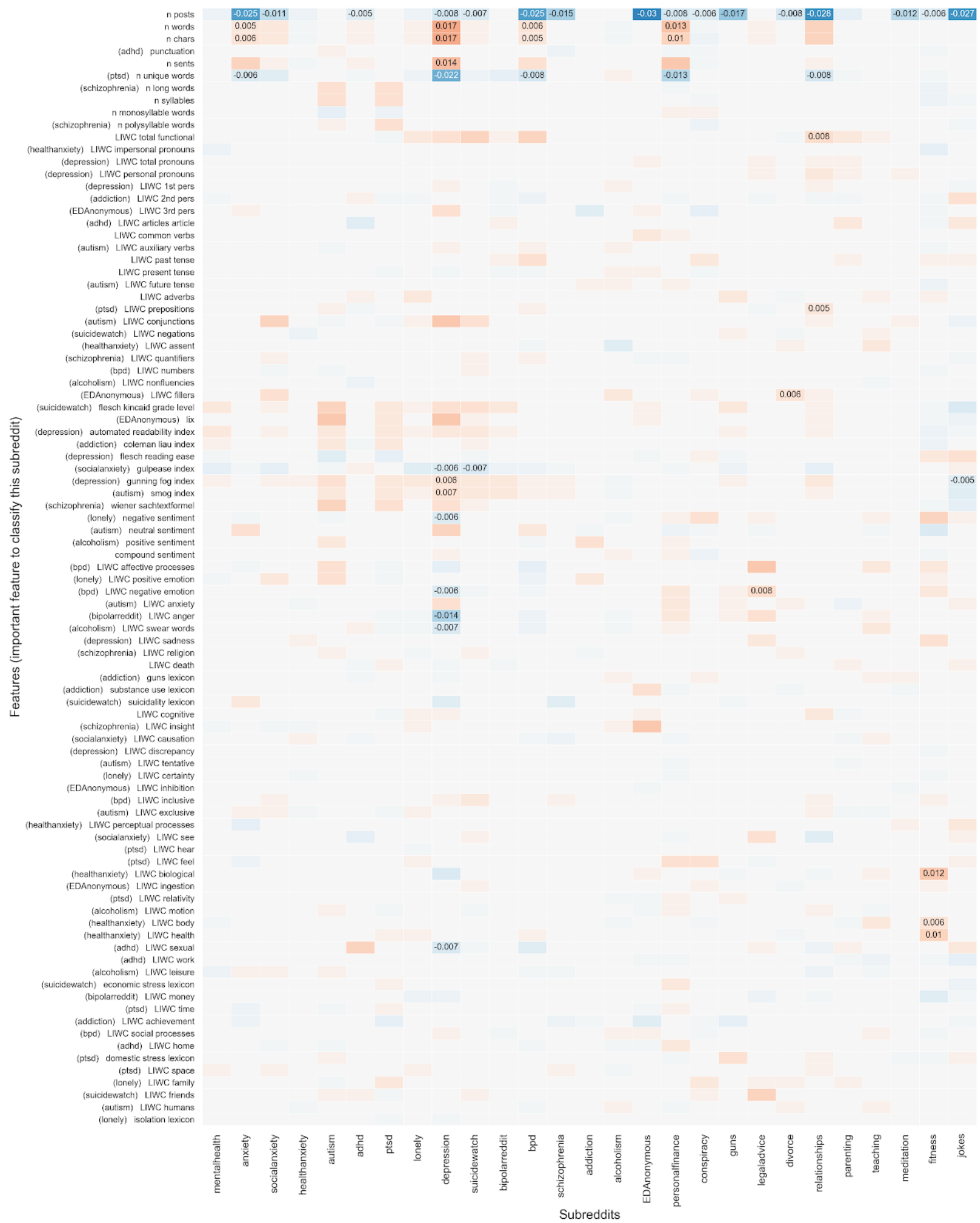

Figure S6. 2019 trend analysis. Trends of mean feature values across subreddits from 2019-03-11 to 2019-03-20. See Figure S5 for more details. 


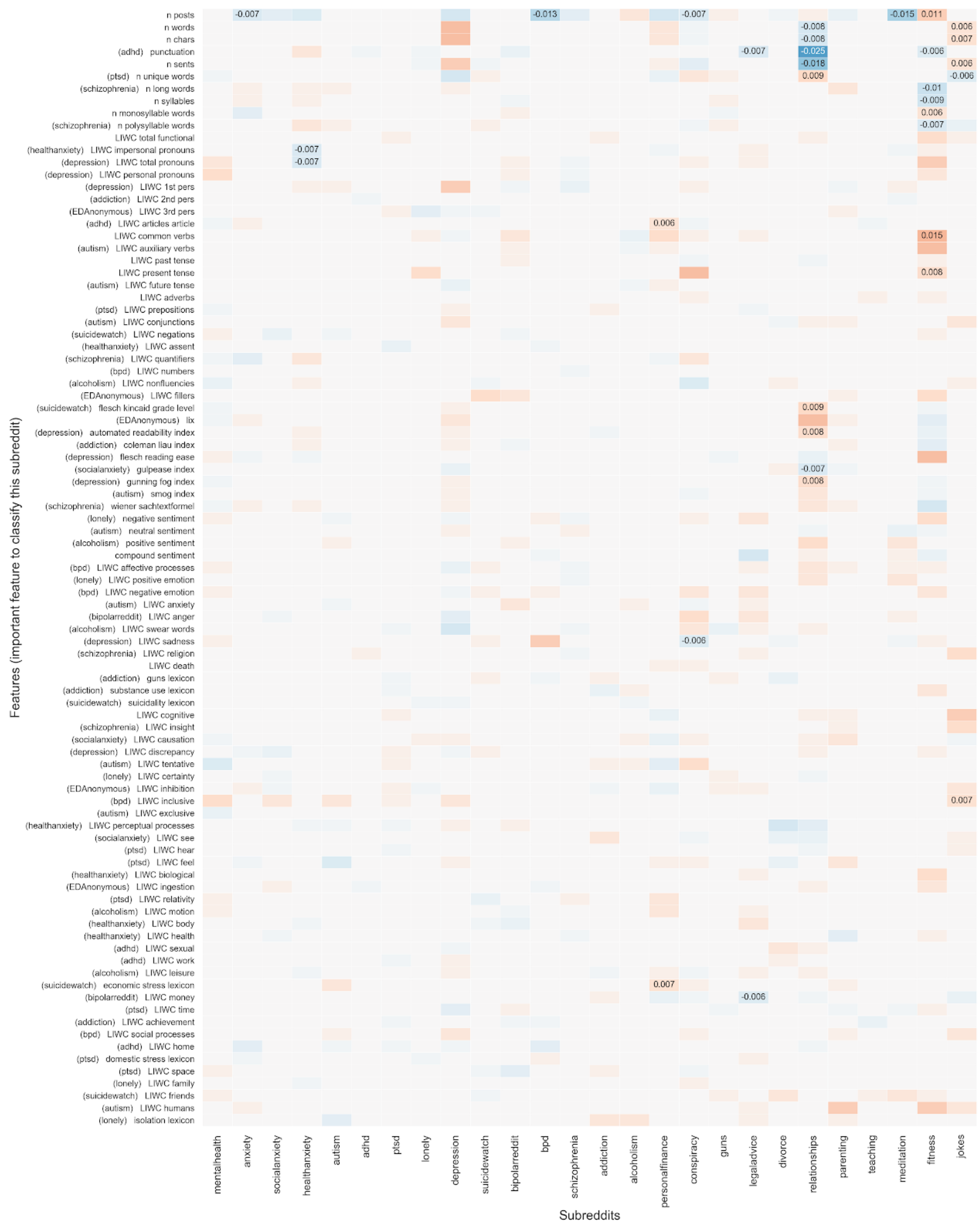

Figure 7. 2018 trend analysis. Trends of mean feature values across subreddits from 2018-03-11 to 2019-03-20. See Figure S5 for more details. 


\subsection{Unsupervised clustering}

\begin{tabular}{|c|c|c|c|c|}
\hline \multirow{2}{*}{$\begin{array}{c}\text { Cluster } \\
\text { Annotation }\end{array}$} & \multicolumn{2}{|r|}{$\begin{array}{c}\text { Distinguishing Features, } \\
\text { Cluster of Pre-Pandemic Posts }\end{array}$} & \multicolumn{2}{|r|}{$\begin{array}{l}\text { Distinguishing Features, } \\
\text { Cluster of Pre-Pandemic Posts }\end{array}$} \\
\hline & $\mathbf{N}$ & Example Features & $\mathbf{N}$ & Example Features \\
\hline Alcohol & 31 & $\begin{array}{l}\text { Substance Use Lexicon total, LIWC } \\
\text { features \{'ingestion', 'inhibition', 'leisure'\}, } \\
\text { TF-IDF features \{'addict', 'alcohol', } \\
\text { 'beer', 'day', 'drank', 'drink', 'drunk', 'everi', } \\
\text { 'last', 'month', 'night', 'problem', 'quit', } \\
\text { 'sober', 'stop', 'week', 'weekend', } \\
\text { 'withdraw'\} }\end{array}$ & 20 & $\begin{array}{l}\text { Substance Use Lexicon total, LIWC features } \\
\text { \{'ingestion', 'leisure'\}, TF-IDF features } \\
\text { \{'alcohol', 'beer', 'day', 'drink', 'drunk', 'everi', } \\
\text { 'night', 'quit', 'sleep', 'sober', 'stop', } \\
\text { 'withdraw'\} }\end{array}$ \\
\hline \begin{tabular}{|l|} 
Alcohol Longer \\
Posts
\end{tabular} & 187 & $\begin{array}{l}\text { Number of characters, long words, } \\
\text { sentences, unique words }\end{array}$ & 128 & $\begin{array}{l}\text { Number of characters, long words, } \\
\text { sentences, unique words, Substance Use } \\
\text { Lexicon total, LIWC features \{'ingestion', } \\
\text { 'inhibition', 'leisure', 'quantifiers', } \\
\text { 'social_processes'\}, TF-IDF features } \\
\text { \{'addict', 'alcohol', 'beer', 'drank', 'drink', } \\
\text { 'drunk', 'problem', 'quit', 'sober', 'stop'\} }\end{array}$ \\
\hline Eating & 18 & $\begin{array}{l}\text { LIWC features \{'biological', 'ingestion'\}, } \\
\text { TF-IDF features \{'ate', 'bing', 'bodi', } \\
\text { 'calori', 'eat', 'ed', 'fat', 'food', 'gain', 'lose', } \\
\text { 'meal', 'purg', 'restrict', 'weight'\} }\end{array}$ & 15 & $\begin{array}{l}\text { LIWC features \{'biological', 'ingestion'\}, } \\
\text { TF-IDF features \{'bing', 'calori', 'eat', 'ed', } \\
\text { 'fat', 'food', 'gain', 'lose', 'purg', 'restrict', } \\
\text { 'weight'\} }\end{array}$ \\
\hline $\begin{array}{l}\text { Eating Longer } \\
\text { Posts }\end{array}$ & 101 & $\begin{array}{l}\text { Numbers of characters, words, and } \\
\text { sentences, LIWC features \{'body', } \\
\text { 'ingestion', 'leisure', 'motion'\}, TF-IDF } \\
\text { features \{'amount', 'ate', 'bing', 'bodi', } \\
\text { 'calori', 'control', 'diet','eat', 'ed', } \\
\text { 'exercis', 'fat', 'food', 'gain', 'pound', } \\
\text { 'purg', 'restrict', 'weight' \} }\end{array}$ & 101 & $\begin{array}{l}\text { Numbers of characters, words, and } \\
\text { sentences, LIWC features \{'body', } \\
\text { 'ingestion', 'leisure', 'motion'\}, TF-IDF } \\
\text { features \{'bing', 'bodi', 'calori', 'control', } \\
\text { 'diet', 'disord', 'eat', 'ed', 'enough', 'fat', } \\
\text { 'food', 'gain', 'lose', 'meal', 'purg', } \\
\text { 'restrict', 'weight' \} }\end{array}$ \\
\hline $\begin{array}{l}\text { Entertain- } \\
\text { ment }\end{array}$ & & & 8 & $\begin{array}{l}\text { Positive Sentiment, Compound Sentiment, } \\
\text { LIWC features \{'leisure', } \\
\text { 'positive_emotion'\}, TF-IDF features } \\
\text { \{'game', 'play', 'video', 'watch'\} }\end{array}$ \\
\hline $\begin{array}{l}\text { General Mental } \\
\text { Health }\end{array}$ & 14 & $\begin{array}{l}\text { LIWC features }\{\text { 'health'\}, TF-IDF features } \\
\{\text { 'health', 'ill', 'mental', 'mental health', } \\
\text { 'mental ill' }\}\end{array}$ & & \\
\hline Health Anxiety & 90 & $\begin{array}{l}\text { LIWC features \{'anxiety', 'biological', } \\
\text { 'body', 'health'\}, TF-IDF features } \\
\text { \{"anxieti', 'arm', 'attack', 'back', 'blood', } \\
\text { 'bodi', 'brain', 'breath', 'cancer', 'caus', } \\
\text { 'check','chest', 'doctor','fear', 'freak', 'googl', } \\
\text { 'head', 'headach', 'health', 'health anxieti', } \\
\text { 'heart', 'pain', 'panic', 'scare', 'side', } \\
\text { 'symptom', 'test', 'weird', 'worri'\} }\end{array}$ & 81 & $\begin{array}{l}\text { LIWC features \{'anxiety', 'biological', } \\
\text { 'body', 'health'\}, TF-IDF features \{"anxieti', } \\
\text { 'attack', 'back', 'blood', 'bodi', 'breath', } \\
\text { 'cancer', 'caus', 'check','chest', 'doctor','fear', } \\
\text { 'freak', 'googl', 'head', 'headach', 'health', } \\
\text { 'health anxieti', 'heart', 'pain', 'panic', 'scare', } \\
\text { 'side', 'symptom', 'test', 'weird', 'worri'\} }\end{array}$ \\
\hline
\end{tabular}




\begin{tabular}{|c|c|c|c|c|}
\hline Loneliness & 28 & $\begin{array}{l}\text { Isolation Lexicon total, LIWC features } \\
\{\text { 'friends', 'sadness', 'social_processes'\}, } \\
\text { TF-IDF features \{'alon', 'feel', 'feel like', } \\
\text { 'friend', 'hang', 'like', 'lone', 'love', 'make', } \\
\text { 'one', 'peopl', 'sad', 'someon', 'talk', 'want'\} }\end{array}$ & 6 & $\begin{array}{l}\text { Flesch Reading Ease, Gulpease Index, } \\
\text { Negative Sentiment, Positive Sentiment, } \\
\text { Isolation Lexicon Total, TF-IDF features } \\
\{\text { ‘lone’\} }\end{array}$ \\
\hline Long Posts & 440 & $\begin{array}{l}\text { Number of: characters, long words, } \\
\text { monosyllabic words, polysyllabic words, } \\
\text { sentences, syllables, unique words }\end{array}$ & 477 & $\begin{array}{l}\text { Number of: characters, long words, } \\
\text { monosyllabic words, polysyllabic words, } \\
\text { sentences, syllables, unique words }\end{array}$ \\
\hline Marijuana & 10 & $\begin{array}{l}\text { TF-IDF features \{'addict', 'drug', 'high', } \\
\text { 'quit', 'smoke', 'smoke weed', 'weed'\} }\end{array}$ & 20 & $\begin{array}{l}\text { TF-IDF features \{'addict', 'drug', 'high', } \\
\text { 'quit', 'smoke', 'smoke weed', 'weed'\} }\end{array}$ \\
\hline Medication & 43 & $\begin{array}{l}\text { LIWC features \{'biological', 'causation', } \\
\text { 'health', 'insight', 'numbers', 'time', 'work'\}, } \\
\text { TF-IDF features \{'day', 'doctor', 'dose', } \\
\text { 'effect', 'experi', 'med', 'medic', 'mg', } \\
\text { 'prescrib', 'side effect', 'start', 'take', 'took', } \\
\text { 'week', 'work'\} }\end{array}$ & 17 & $\begin{array}{l}\text { LIWC features \{'causation', 'health'\}, } \\
\text { TF-IDF features \{'effect', 'med', 'medic', } \\
\text { 'side', 'side effect','take'\} }\end{array}$ \\
\hline Panic Attacks & 9 & $\begin{array}{l}\text { Negative sentiment, LIWC features } \\
\text { \{affective processes, anger, anxiety, } \\
\text { negative emotion\}, TF-IDF } \\
\text { features \{'anxieti', 'attack', 'panic', 'panic } \\
\text { attack'\} }\end{array}$ & 13 & $\begin{array}{l}\text { Negative sentiment, LIWC features } \\
\text { \{affective processes, anger, anxiety, body, } \\
\text { negative emotion\}, TF-IDF } \\
\text { features \{'anxieti', 'attack', 'breath', 'panic', } \\
\text { 'panic attack'\} }\end{array}$ \\
\hline Pleading & 12 & $\begin{array}{l}\text { TF-IDF features \{'help', 'please', 'cant', } \\
\text { 'dont', 'dont know', 'know','im', 'ive'\}, } \\
\text { Flesch Reading Ease, Gulpease Index }\end{array}$ & 12 & $\begin{array}{l}\text { TF-IDF features \{'anymor','cant', 'dont', } \\
\text { 'dont know', 'know','im', 'ive', 'want'\}, } \\
\text { Flesch Reading Ease, Gulpease Index, } \\
\text { Suicidality Lexicon Total, LIWC features } \\
\text { \{'death'\} }\end{array}$ \\
\hline Resources & 17 & $\begin{array}{l}\text { TF-IDF features \{'com', 'https', 'https } \\
\text { www', 'share', 'www'\} }\end{array}$ & 16 & $\begin{array}{l}\text { TF-IDF \{'com', 'help', 'https', 'https www', } \\
\text { 'www'\} }\end{array}$ \\
\hline Resources & 12 & $\begin{array}{l}\text { TF-IDF features \{'com', 'https', 'https } \\
\text { www', 'www'\} }\end{array}$ & 12 & $\begin{array}{l}\text { TF-IDF features \{'com', 'https', 'https www', } \\
\text { 'www'\} }\end{array}$ \\
\hline Resources & & & 7 & $\begin{array}{l}\text { TF-IDF features \{'https', 'https www', } \\
\text { 'www'\} }\end{array}$ \\
\hline Seeking Advice & 13 & $\begin{array}{l}\text { Neutral Sentiment, Compound Sentiment, } \\
\text { TF-IDF features \{'advic', 'ani', 'ani advic', } \\
\text { 'ani tip', 'anyon', 'anyon ani', 'appreci', 'doe', } \\
\text { 'doe anyon', 'help', 'tip'\} }\end{array}$ & & \\
\hline $\begin{array}{l}\text { Seeking } \\
\text { Normali- } \\
\text { zation }\end{array}$ & 7 & $\begin{array}{l}\text { Coleman Liau index, Neutral Sentiment, } \\
\text { TF-IDF features \{'anyon', 'anyon els', 'doe', } \\
\text { 'doe anyon', 'els'\} }\end{array}$ & 8 & $\begin{array}{l}\text { Coleman Liau index, Neutral Sentiment, } \\
\text { TF-IDF features \{'anyon', 'anyon els', 'doe', } \\
\text { 'doe anyon', 'els'\}, Automated Readability } \\
\text { Index }\end{array}$ \\
\hline Self Harm & & & 11 & $\begin{array}{l}\text { Negative Sentiment, Suicidality Lexicon } \\
\text { Total, LIWC features \{'affective_processes', } \\
\text { 'death', 'humans', 'negative_emotion'\}, } \\
\text { TF-IDF features \{'harm', 'self', 'self harm', } \\
\text { 'suicid'\} }\end{array}$ \\
\hline Social Anxiety & 13 & TF-IDF features \{'anxieti', 'awkward', & 15 & LIWC features \{'social_porcesses'\}, TF-IDF \\
\hline
\end{tabular}




\begin{tabular}{|c|c|c|c|c|}
\hline & & 'peopl', 'social', 'social anxieti', 'talk'\} & & $\begin{array}{l}\text { features \{'anxieti', 'anxious', 'awkward', } \\
\text { 'peopl', 'social', 'social anxieti', 'talk'\} }\end{array}$ \\
\hline Suicidality & 23 & $\begin{array}{l}\text { Sentiment Negative, Suicidality lexicon } \\
\text { total, LIWC features \{'anger', 'death', } \\
\text { 'negative_emotion', 'sadness', 'sexual', } \\
\text { 'swear_words'\}, TF-IDF features } \\
\text { \{'anymor', 'die', 'end', 'fuck', 'hate', 'kill', } \\
\text { 'life', 'live', 'noth', 'shit', 'suicid', 'want', } \\
\text { 'want die'\} }\end{array}$ & 349 & $\begin{array}{l}\text { Number of characters, words, sentences; } \\
\text { Totals for the COVID-19, Economic Stress, } \\
\text { Isolation, Domestic Stress and Suicidality } \\
\text { Lexicons; LIWC features \{'anger', 'anxiety', } \\
\text { 'death','family', 'friends', 'future_tense', } \\
\text { 'home', 'money', 'motion', 'negative_emotion', } \\
\text { 'sadness', 'sexual', 'social_processes', } \\
\text { 'swear_words', 'work'\}, TF-IDF features } \\
\text { ‘'abus', 'anymor', 'college', 'die', 'end', } \\
\text { 'fuck', 'hate', 'kill', 'life', 'live', 'noth', 'shit', } \\
\text { 'suicid', 'want'\} }\end{array}$ \\
\hline Unspecified & 9 & $\begin{array}{l}\text { Automated readability index, Coleman } \\
\text { Liau index, Flesch Kincaid Grade Level, } \\
\text { Gunning Fog Index, LIX, Smog Index, } \\
\text { Wiener Sachtextformel, Neutral Sentiment, } \\
\text { Compound Sentiment }\end{array}$ & 22 & $\begin{array}{l}\text { Automated readability index, Coleman Liau } \\
\text { index, Flesch Kincaid Grade Level, Gunning } \\
\text { Fog Index, LIX, Smog Index, Wiener } \\
\text { Sachtextformel, Neutral Sentiment, } \\
\text { Compound Sentiment, COVID-19 Lexicon } \\
\text { Total, TF-IDF features \{'advic', 'ani', } \\
\text { 'diagnos', 'diagnosi', 'experi', 'med', } \\
\text { 'medic', 'tip'\} }\end{array}$ \\
\hline Unspecified 2 & 350 & $\begin{array}{l}\text { Automated readability index, } \\
\text { Flesch-Kincaid grade level, Gunning fog } \\
\text { index, Smog index, } \\
\text { Numbers of words, syllables, and } \\
\text { sentences, LIWC features \{'causation', } \\
\text { 'family', 'home', 'insight', 'leisure', } \\
\text { 'money', 'religion', 'sexual'\} }\end{array}$ & & \\
\hline
\end{tabular}

Table S4. Cluster annotations. Cluster annotations were assigned based on a review of the features found to distinguish each cluster, using Wilcoxon rank-sum tests with Bonferroni correction. Representative significantly cluster-associated features informing the annotation are shown, along with the total number of significant features per cluster. Clustering was performed separately with $\mathrm{k}=20$ on the pre-pandemic posts dataset from 2019 and the mid-pandemic posts dataset from 2020. The majority of clusters were approximately replicated between the two time periods. Each pair of clusters (one from the pre-pandemic data and one from the mid-pandemic data) assigned the same annotation are shown side-by-side to illustrate the overlap of their predictive features. A few clusters were detected only in the pre-pandemic dataset (e.g., General Mental Health, Seeking Advice) and a few were only detected in the mid-pandemic dataset (e.g., Self Harm, Entertainment). Two "Resources" clusters were detected in the pre-pandemic dataset, and three were detected in the mid-pandemic dataset. The characteristic features were not meaningfully distinct for any among these "Resources" clusters and these clusters partitioned an island of posts visible in UMAP space, so all "Resources" clusters detected in a given dataset were collapsed into a single cluster. 


\begin{tabular}{|c|c|c|c|}
\hline \multirow[b]{2}{*}{$\begin{array}{c}\text { Cluster } \\
\text { Annotation }\end{array}$} & \multicolumn{3}{|c|}{$\begin{array}{c}\text { Attributes for } \\
\text { Cluster of Pre-Pandemic Posts }\end{array}$} \\
\hline & $\begin{array}{c}\text { Diversity } \\
\text { (Shannon Index) }\end{array}$ & $\begin{array}{c}\text { Size } \\
\text { (Number of Posts) }\end{array}$ & Mean Post Length \\
\hline Eating Longer Posts & 1.09 & 218 & 364.08 \\
\hline Health Anxiety & 1.30 & 1105 & 193.50 \\
\hline Social Anxiety & 1.37 & 421 & 107.62 \\
\hline Eating & 1.42 & 1009 & 108.04 \\
\hline Alcohol & 1.67 & 1067 & 155.89 \\
\hline Alcohol Longer Posts & 2.08 & 238 & 553.95 \\
\hline Marijuana & 2.10 & 246 & 161.64 \\
\hline Suicidality & 2.63 & 1438 & 112.94 \\
\hline Loneliness & 2.67 & 1620 & 121.41 \\
\hline Panic Attacks & 2.76 & 413 & 150.87 \\
\hline Medication & 2.87 & 412 & 176.71 \\
\hline Resources & 3.56 & 490 & 118.88 \\
\hline $\begin{array}{l}\text { General Mental } \\
\text { Health }\end{array}$ & 3.59 & 416 & 135.82 \\
\hline Seeking Advice & 3.61 & 618 & 92.71 \\
\hline Long Posts & 3.67 & 534 & 885.73 \\
\hline Unspecified 2 & 3.71 & 4272 & 318.10 \\
\hline \begin{tabular}{|l|} 
Seeking \\
Normalization \\
\end{tabular} & 3.72 & 739 & 71.50 \\
\hline Pleading & 3.74 & 915 & 81.22 \\
\hline Unspecified & 3.78 & 6329 & 94.32 \\
\hline
\end{tabular}

Table S5. Cluster attributes on pre-pandemic posts. Cluster attributes, including total cluster size in number of posts, mean post length, and diversity are shown for each cluster from the pre-pandemic post dataset. Diversity was computed with the scikit-bio package of alpha diversity measures using the Shannon Diversity Index based on the representation within the cluster of posts across the 15 subreddits included in the analysis. Higher values indicate greater diversity. Clusters are sorted in ascending order according to their diversity. 

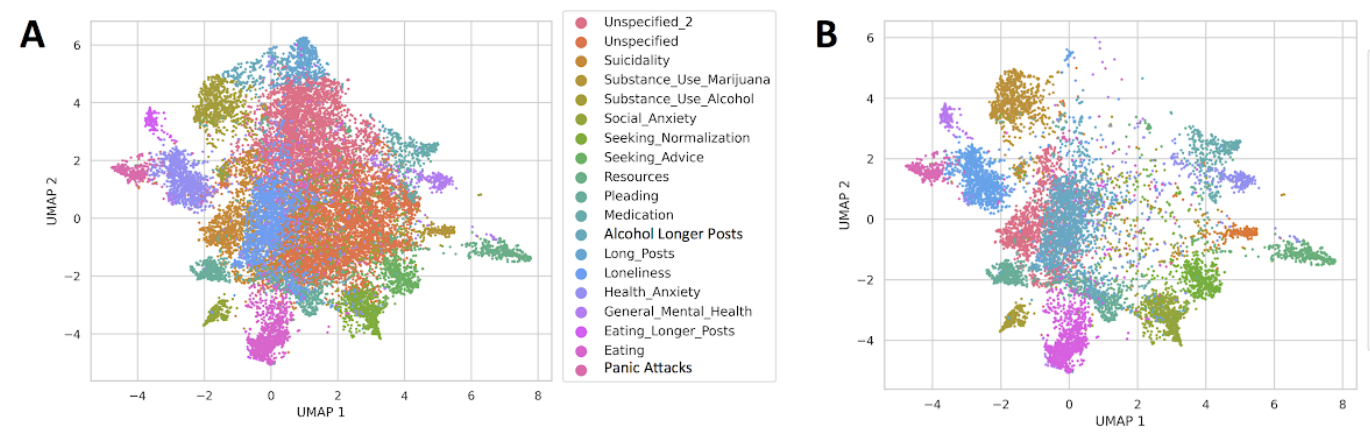

- Suicidality

Substance Use Alcohol

- Social_Anxiety

Seeking_Normalization

- Seeking_Advice

Resources

- Pleading

Medication

Loneliness

Health_Anxiety

- Eating_longer_Posts

- Eating_Loros

Panic Attacks
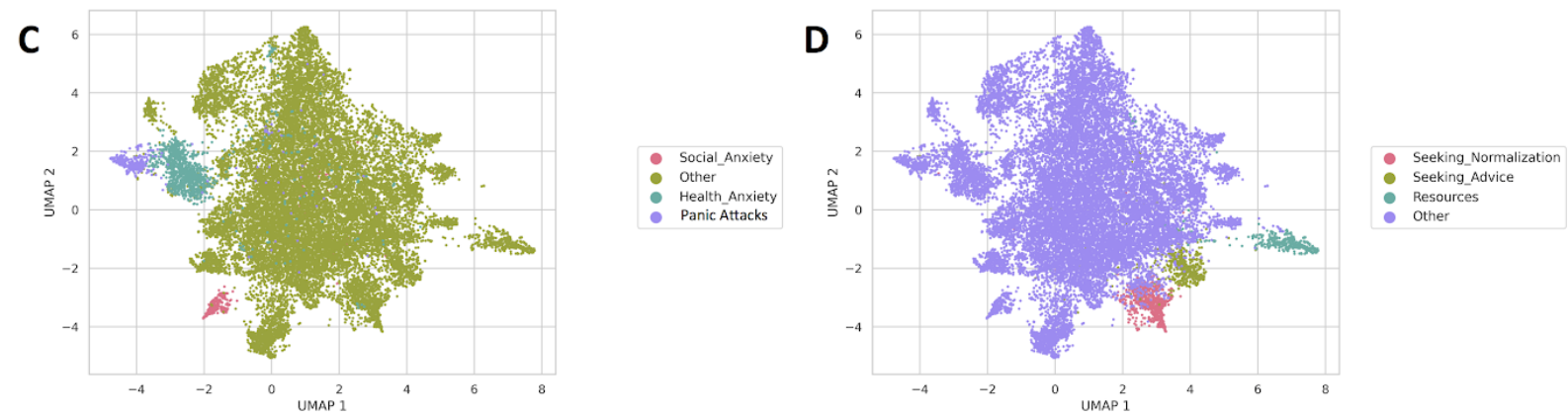

Figure S8. Unsupervised clustering of posts made in 2019 to 15 mental health subreddits. Values of k from 5 to 50 were tested in increments of five and the quality of the resulting graph partitions were evaluated with a modularity metric. Modularity scores rose to a plateau of approximately 0.65 for $\mathrm{k} \geq 10$ and $\mathrm{k}=20$ was selected because it yielded sizable clusters with more interpretable cluster-distinguishing features than $\mathrm{k}=10$ or 15 . A) UMAP with all clusters displayed with equal point translucency. B) UMAP with the two largest, and the two containing the longest posts, removed to highlight proximities among the remaining clusters. C) Highlighting the proximity of anxiety-related clusters. D) Highlighting the proximity of clusters related to resource provision and advice seeking. 
A

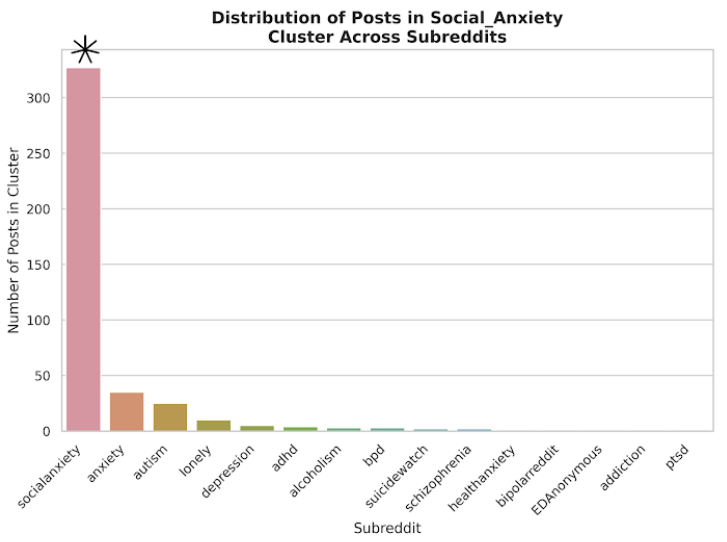

C

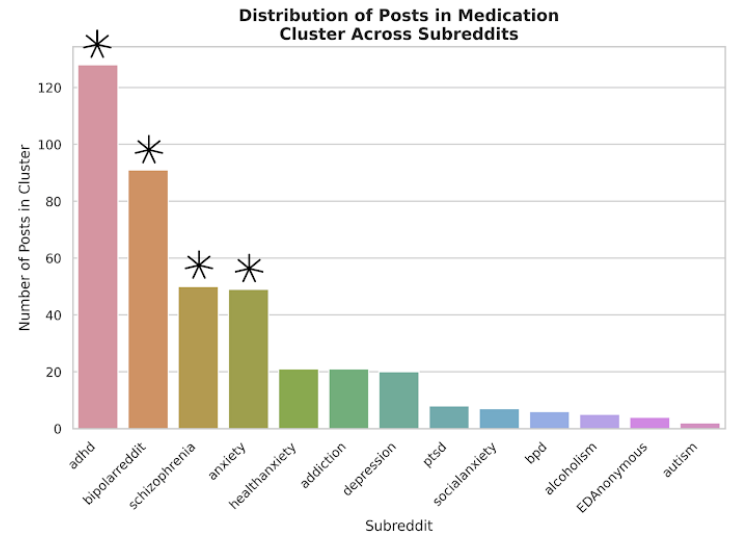

B

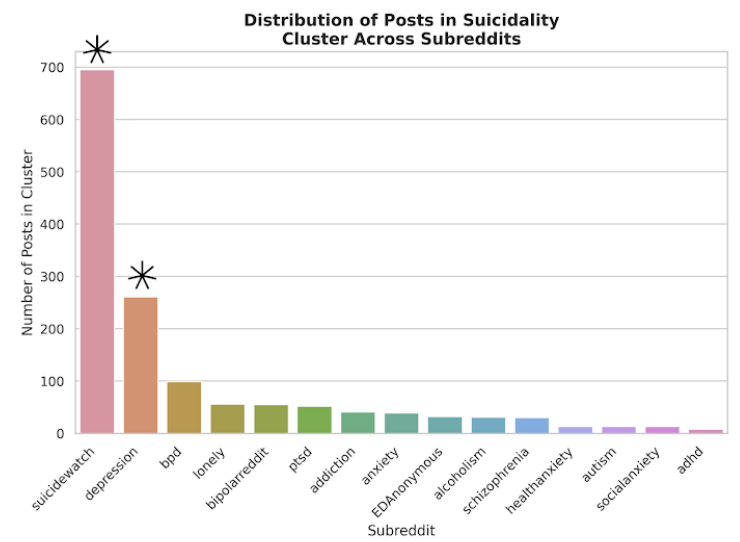

D

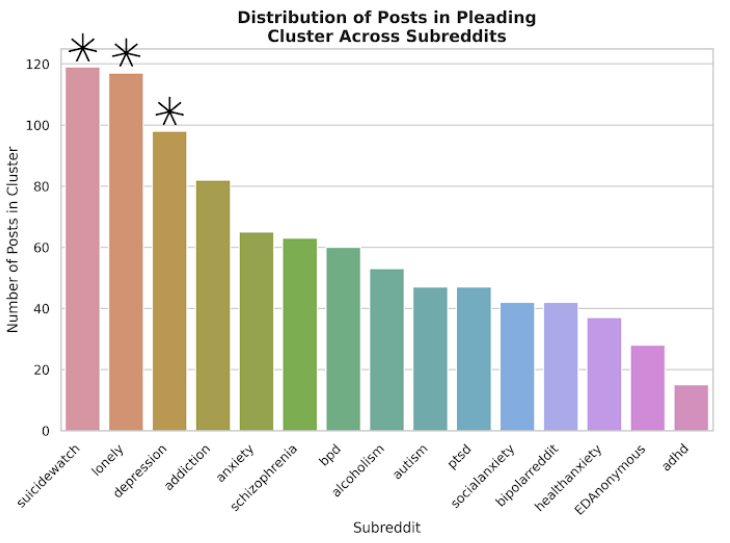

Figure S9. Representation of posts from each subreddit within a given cluster for the 2019 pre-pandemic dataset.

A) the Social Anxiety cluster, B) the Suicidality cluster, C) the Medication cluster, D) and the Pleading cluster. Columns are marked with stars for subreddits on which posts from the given cluster were significantly enriched. See Figure 3B for the full set of cluster enrichments on the analysed subreddits.

\subsection{Topic Modeling with Latent Dirichlet Allocation (LDA)}

We analyzed whether the subreddits that most increased in their Health Anxiety topic correlated with the ones that most increased in negative semantic change as measured by the trend analysis, but this was not significant $(\rho=$ -0.046, $P=0.819$ ). 


\begin{tabular}{|l|l|}
\hline Topic & Words \\
\hline $\begin{array}{l}\text { Autism/ADHD + } \\
\text { School/Work }\end{array}$ & autism, work, help, adhd, school, people, autist, https, time, need \\
\hline Alcohol/Addiction & year, addic, time, go, know, month, help, drink, want, work \\
\hline Sleep Issues & go, sleep, night, fuck, time, hour, tell, come, say, know \\
\hline Alcohol/Eating Disorders & drink, eat, weight, alcohol, food, feel, bing, lose, week, know \\
\hline Social Interaction & friend, peopl, talk, feel, know, social, want, think, time, school \\
\hline Schizophrenia & think, thing, feel, know, experi, hear, peopl, voic, thought, schizophrenia \\
\hline Medical/Medication & medic, take, depress, med, feel, bipolar, doctor, help, start, work \\
\hline Health Anxiety & anxieti, feel, go, attack, panic, pain, hert, start, doctor, week \\
\hline Mental Health Help & help, know, tell, mental, year, say, want, thing, diagnos, think \\
\hline Life & feel, want, life, know, think, love, time, thing, go, peopl \\
\hline
\end{tabular}

Table S6: Pre-Pandemic Topic Model. Topics extracted from LDA on pre-pandemic mental health subreddits. 


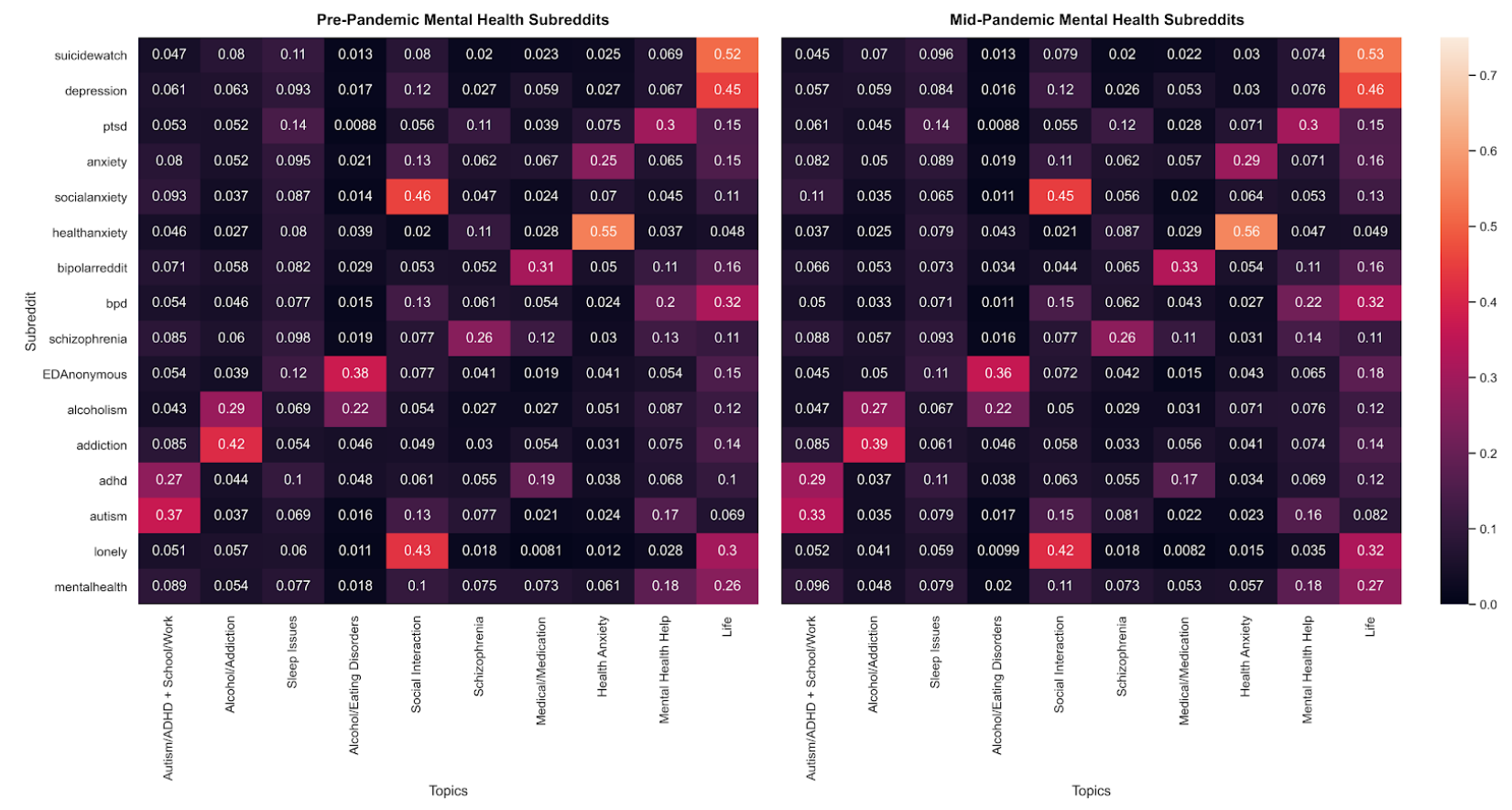

Figure S10: Pre-pandemic LDA model distribution over mental health subreddits. Distribution of pre-pandemic LDA topics for posts in mental health subreddits pre-pandemic (left) and mid-pandemic (right). This highlights that the distributions of these particular topics within a single subreddit largely did not change between pre- and mid-pandemic timeframes, except for an increase in the topics "Health Anxiety" and "Life" and a decrease in the "Alcohol/Addiction" topic.

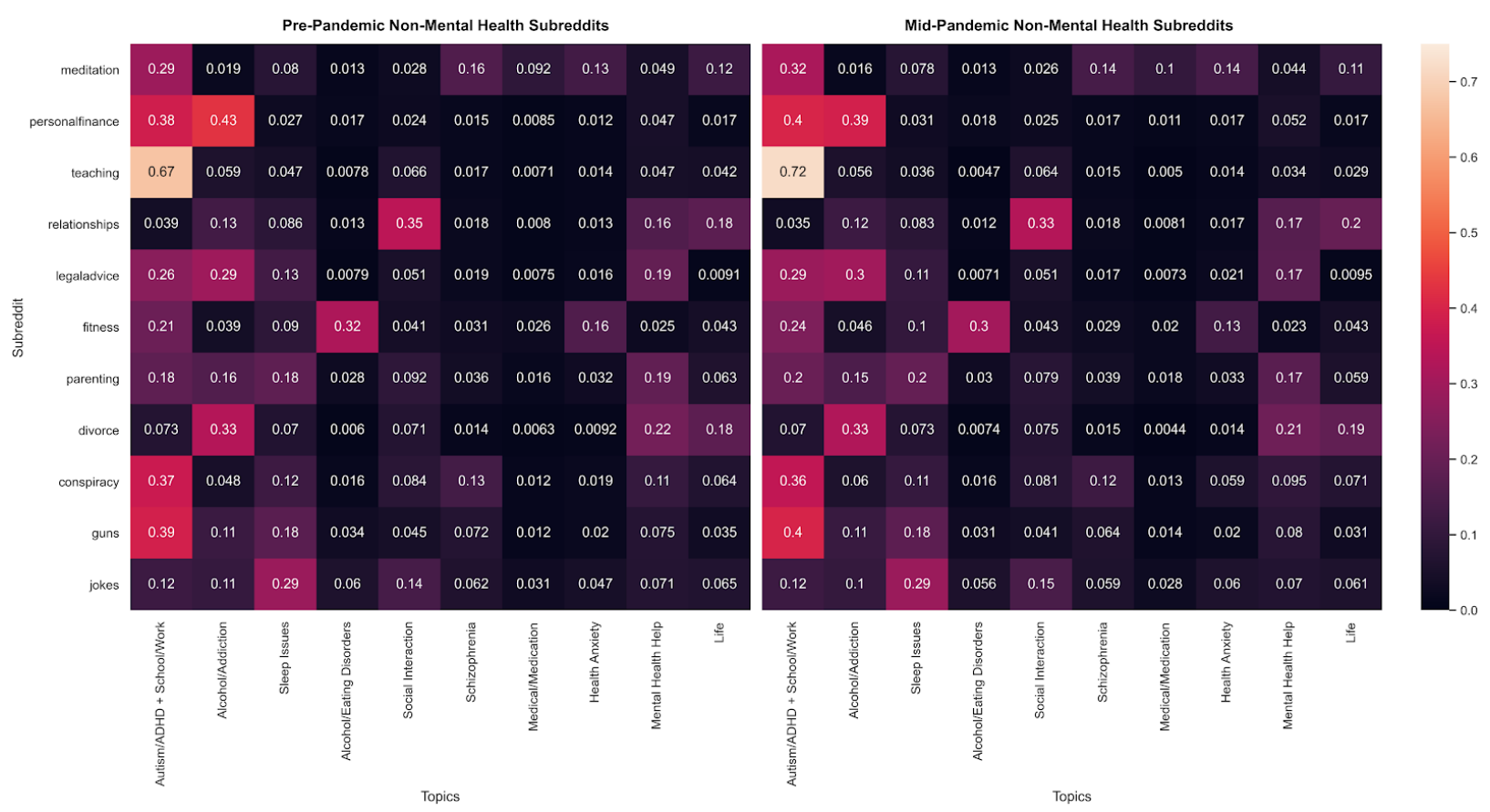

Figure S11: Pre-pandemic LDA model over non-mental health subreddits. Distribution of pre-pandemic LDA topics for posts in non-mental health subreddits pre-pandemic (left) and mid-pandemic (right). As with the mental 
health subreddits, distributions of these particular topics within a single subreddit largely did not change between pre- and mid-pandemic timeframes, except for an increase in the topics "Health Anxiety" and "Life" and a decrease in the "Alcohol/Addiction" topic. 


\subsection{Measuring Similarity Between Subreddits over Time with Supervised Dimensionality Reduction}
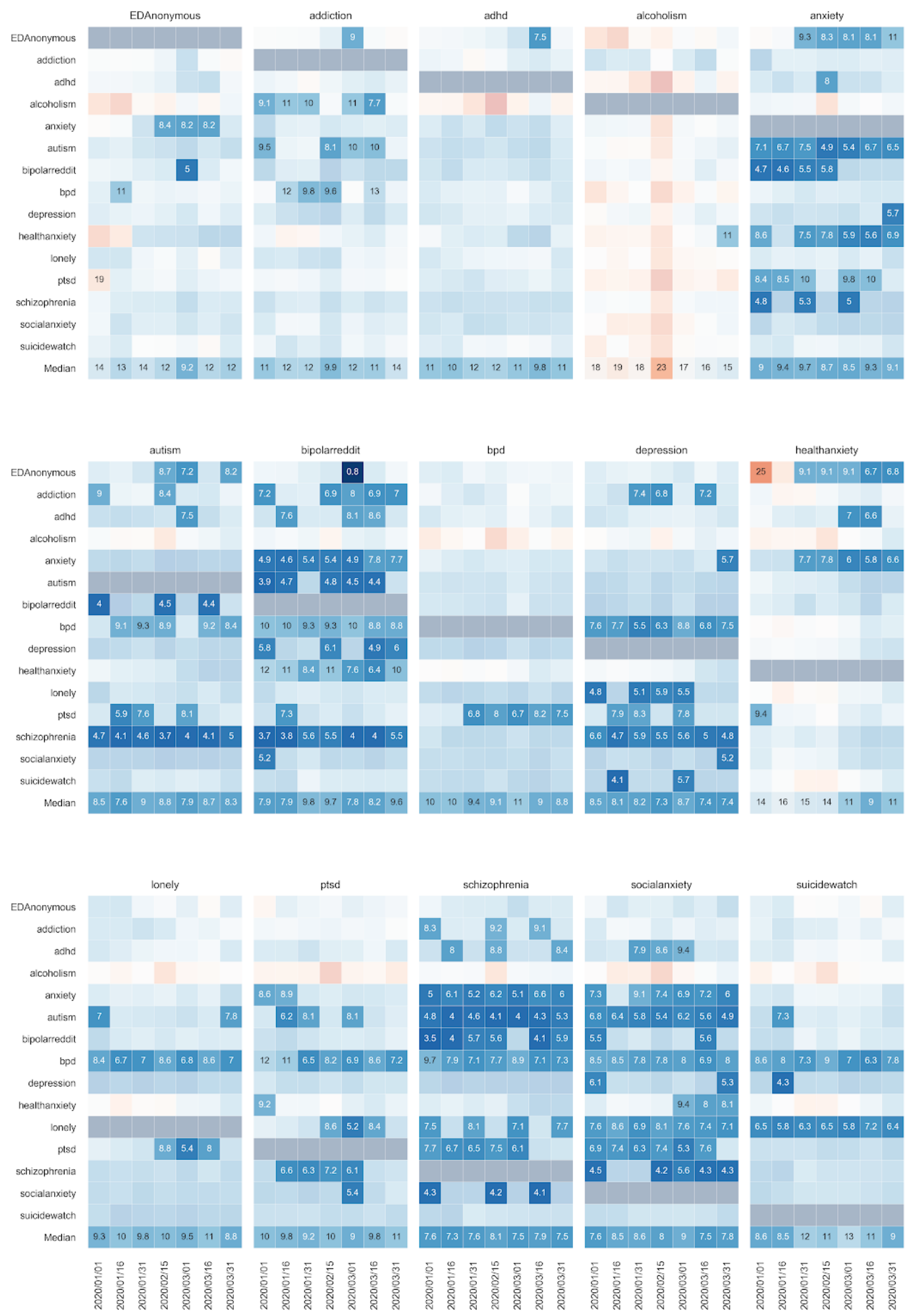

Figure S12. Pairwise changes in distance between subreddits throughout pandemic. Total pairwise distance between subreddits for each time window displaying only extreme values with regards to normal 2019 fluctuations 
(top and bottom 5th percentiles), which indicates they are less likely to be part of regular fluctuations in distance (see Supplement 1.4 for a precision analysis of this approach).

\section{References}

1. Hutto CJ, Gilbert E. Vader: A parsimonious rule-based model for sentiment analysis of social media text. Proceedings of the 8th International AAAI Conference on Weblogs and Social Media 2014. Available from: https://www.aaai.org/ocs/index.php/ICWSM/ICWSM14/paper/viewPaper/8109

2. DeWilde B. textacy Documentation. 2017. Available from:

https://buildmedia.readthedocs.org/media/pdf/textacy/latest/textacy.pdf

3. Pennebaker JW, Booth RJ, Boyd RL, Francis ME. LIWC 2015 Operator's Manual. Austin, TX: Pennebaker Conglomerates Inc; 2015. 